Алгебра и анализ

Том. 16 (2004), вып. 1
St. Petersburg Math. J.

Vol. 16 (2005), No. 1, Pages 211-236

S 1061-0022(04)00848-9

Article electronically published on December 17, 2004

\title{
PINNING OF MAGNETIC VORTICES BY AN EXTERNAL POTENTIAL
}

\author{
I. M. SIGAL AND F. TING
}

Dedicated to M. Sh. Birman with admiration and friendship

\begin{abstract}
The existence and uniqueness of vortex solutions is proved for GinzburgLandau equations with external potentials in $\mathbb{R}^{2}$. These equations describe the equilibrium states of superconductors and the stationary states of the $U(1)$-Higgs model of particle physics. In the former case, the external potentials are due to impurities and defects. Without the external potentials, the equations are translationally (as well as gauge) invariant, and they have gauge equivalent families of vortex (equivariant) solutions called magnetic or Abrikosov vortices, centered at arbitrary points of $\mathbb{R}^{2}$. For smooth and sufficiently small external potentials, it is shown that for each critical point $z_{0}$ of the potential there exists a perturbed vortex solution centered near $z_{0}$, and that there are no other single vortex solutions. This result confirms the "pinning" phenomena observed and described in physics, whereby magnetic vortices are pinned down to impurities or defects in the superconductor.
\end{abstract}

\section{§1. IntRoduCtion AND STATEMENT OF THE PROBLEM}

For a superconductor of type II, if the external magnetic field satisfies $h_{c}<h<h_{c_{2}}$, then the magnetic field penetrates the material in tubular flux lines called magnetic vortices. To date, superconductors have been very useful in making steady magnetic fields of over 100,000 Gauss. One major problem encountered when trying to produce large magnetic fields is the dissipation of energy due to creeping or flow of vortices [T]. The phenomenon of creeping of vortices can be explained by the Lorentz force between the superconducting current and the magnetic flux lines. It can be shown that the force acting on a single vortex is proportional to the superconducting current. This force moves the flux lines transversely to the current, which, in turn, induces an electric field parallel to the current. The electric field generated by the moving vortex acts to resist the current flow, and hence energy is dissipated.

A way to resolve the problem of creeping of vortices is to pin vortices down. It is well known that spatial inhomogeneities, impurities or point defects in the sample, or variable thickness $[\overline{D G}]$ in the superconducting material can immobilize a flux line. Showing that this indeed happens, say in the framework of the macroscopic model of a superconductor, leads to an interesting mathematical problem. Namely, the problem is to prove that out of a continuum of vortex states only a few survive when an impurity potential is introduced, and that the surviving states are localized near critical points of the potential in question. Moreover, one would like to determine which of these states are

2000 Mathematics Subject Classification. Primary 58E50; Secondary 35B20, 82D55, 35Q55, 35Q60.

Key words and phrases. Superconductivity, Ginzburg-Landau equations, pinning, magnetic vortices, external potential, existence.

Supported by NSERC (grant N7901). 
stable. The first of these problems is addressed in this paper, while the second (stability of vortex states) will be addressed elsewhere [ST].

In this paper we work within the standard macroscopic (or mean field) theory of superconductivity. In this theory, due to Ginzburg and Landau, the stationary states of superconductors are described by pairs $(\psi, A)$, where $\psi: \mathbb{R}^{2} \rightarrow \mathbb{C}$ is the order parameter and $A: \mathbb{R}^{2} \rightarrow \mathbb{R}^{2}$ is the magnetic potential. These states satisfy the system of equations

$$
\begin{aligned}
-\Delta_{A} \psi+\frac{\lambda}{2}\left(|\psi|^{2}-1\right) \psi & =0, \\
\nabla \times \nabla \times A+\operatorname{Im}\left(\bar{\psi} \nabla_{A} \psi\right) & =0,
\end{aligned}
$$

called the Ginzburg-Landau (GL) equations. Here $\lambda>0$ is a constant depending on the material in question, $\nabla_{A}=\nabla-i A$ is the covariant gradient, and $\Delta_{A}=\nabla_{A}^{2}$. For a vector $A, \nabla \times A$ is the scalar $\partial_{1} A_{2}-\partial_{2} A_{1}$, and for a scalar $\xi, \nabla \times \xi$ is the vector $\left(-\partial_{2} \xi, \partial_{1} \xi\right)$. Equation (1.2) is the Maxwell equation involving the magnetic field $B=\operatorname{curl} A$ and the supercurrent $\operatorname{Im}\left(\bar{\psi} \nabla_{A} \psi\right)$. Equations (1.1) and (1.2) are supplemented with the boundary condition

$$
|\psi(x)| \rightarrow 1 \quad \text { as }|x| \rightarrow \infty .
$$

Equations (1.1) and (1.2) are the Euler-Lagrange equations for the Ginzburg-Landau energy functional

$$
\mathcal{E}(\psi, A)=\frac{1}{2} \int_{R^{2}}\left(\left|\nabla_{A} \psi\right|^{2}+(\nabla \times A)^{2}+\frac{\lambda}{4}\left(|\psi|^{2}-1\right)^{2}\right) d x
$$

i.e., the solutions of (1.1) and (1.2) are critical points of $\mathcal{E}: \mathcal{E}^{\prime}(\psi, A)=0$.

We define the vorticity or the winding number of a vector field $\psi: \mathbb{R}^{2} \rightarrow \mathbb{C}$ at infinity as $\operatorname{deg} \psi:=\operatorname{deg}\left(\left.\frac{\psi}{|\psi|}\right|_{|x|=R}\right)=\frac{1}{2 \pi} \int_{|x|=R} d(\arg \psi)$ for $R$ sufficiently large. Assume that a pair $(\psi, A)$ has finite energy; then the degree of the vector field $\psi$ is related to the flux of the magnetic field $B=\operatorname{curl} A$ as follows:

$$
\int_{\mathbb{R}^{2}} B d x=2 \pi(\operatorname{deg} \psi)
$$

Besides the trivial solutions $\psi=1, A=0$ and $\psi=0, \nabla \times A=B_{0}\left(B_{0}\right.$ is a constant vector field), which correspond to purely superconducting and normal states of the material, respectively, equations (1.1)-(1.2) also have remarkable solutions of the form

$$
\psi_{n}(x)=f_{n}(r) e^{i n \theta} \quad \text { and } \quad A_{n}(x)=a_{n}(r) \nabla(n \theta)
$$

called (magnetic or Abrikosov) $n$-vortices. Here $(r, \theta)$ are the polar coordinates of the vector $x \in \mathbb{R}^{2}$ and $n$ is an integer. Note that $\operatorname{deg} \psi_{n}=n$. The existence of such solutions was predicted by A. Abrikosov, who together with V. L. Ginzburg shared the 2003 Nobel prize in Physics for this discovery (see www.nobel.se). The existence was proved by Pholr $[\mathrm{P}]$ and by Berger and Chen $[\mathrm{BC}]$ by using methods of the calculus of variations. Stability of $n$-vortices was proved by Gustafson and Sigal GS1.

The following information on the vortex profiles $f_{n}$ and $a_{n}$ is available (see $[\mathrm{BC}]$ ): $0<f_{n}<1,0<a_{n}<1$ on $(0, \infty) ; f_{n}^{\prime}, a_{n}^{\prime}>0 ; 1-f_{n}, 1-a_{n} \rightarrow 0$ as $r \rightarrow \infty$ with an exponential rate of decay; $f_{n} \approx c r^{n}, a_{n} \approx d r^{2}(c>0, d>0$ are constants) as $r \rightarrow 0$.

Equations (1.1) and (1.2) have translational and gauge symmetries

$$
\psi(x) \mapsto \psi(x-z), \quad A(x) \mapsto A(x-z)
$$

and

$$
\psi \mapsto e^{i \gamma} \psi, \quad A \mapsto A+\nabla \gamma
$$


for any $z \in \mathbb{R}^{2}$ and any twice differentiable $\gamma: \mathbb{R}^{2} \rightarrow \mathbb{R}$, respectively. Consequently, solutions (1.4) lead to the following families of solutions:

$$
\psi_{n z \gamma}(x)=e^{i \gamma} \psi_{n}(x-z), \quad A_{n z \gamma}(x)=A_{n}(x-z)+\nabla \gamma(x-z),
$$

where $n$ is an integer, $z \in \mathbb{R}^{2}$ and $\gamma: \mathbb{R}^{2} \rightarrow \mathbb{R}$.

Equations (1.1)-(1.2) also have rotational symmetry:

$$
\psi(x) \rightarrow \psi(g x), \quad A(x) \rightarrow g^{-1} A(g x)
$$

for any $g \in O(2)$, but this symmetry plays no role in our analysis.

If a superconductor has impurities, then the GL equations are modified to

$$
\begin{aligned}
-\Delta_{A} \psi+\frac{\lambda}{2}\left(|\psi|^{2}-1\right) \psi+W_{\epsilon}(x) \psi & =0, \\
\nabla \times \nabla \times A+\operatorname{Im}\left(\bar{\psi} \nabla_{A} \psi\right) & =0,
\end{aligned}
$$

where $W_{\epsilon}: \mathbb{R}^{2} \rightarrow \mathbb{R}$ is a potential of impurities. We assume that $W_{0}=0$.

The problem we address in this paper is the existence and uniqueness of vortex-type solutions of the Ginzburg-Landau system (1.6)-(1.7) with external potential $W_{\epsilon}$, near the vortex solutions (1.5).

Notation. Throughout this paper, we are working with Sobolev spaces but we use only $L^{2}$ inner products.

Acknowledgement. I.M.S. thanks S. Gustafson for fruitful collaboration on magnetic vortices.

\section{$\S 2$. Results}

In this section, we state our assumptions on the potential $W_{\epsilon}$ and our main theorems. In what follows, we consider only the 1-vortex $\left(\psi_{1}, A_{1}\right)$ and use the notation $\psi_{0} \equiv \psi_{1}$ and $A_{0} \equiv A_{1}$. Results for the -1 -vortex are exactly the same. Proving similar results for $n$-vortices requires some additional technical steps and the corresponding results will be presented elsewhere.

We assume the potential $W_{\epsilon}$ satisfies the following conditions:

- (A) $W_{\epsilon}$ is $O(\epsilon)$ in $L^{2}$;

- (B) $\left|\partial_{x}^{\alpha} W_{\epsilon}(x)\right| \leq C_{\alpha} \epsilon \delta^{|\alpha|}$ for $0 \leq|\alpha| \leq m$ and for some $\epsilon, \delta>0$.

Define the effective potential experienced by the vortex $\left(\psi_{0}, A_{0}\right)$ as

$$
W_{\mathrm{eff}, \epsilon}(z)=\frac{1}{2} \int W_{\epsilon}(x)\left(\left|\psi_{0}(x-z)\right|^{2}-1\right) d x .
$$

We consider the domain

$\Omega_{\epsilon \delta}=\left\{x \in \mathbb{R}^{2}\right.$ : if $\left|W_{\epsilon}^{\prime}(x)\right| \ll \epsilon \delta$, then $W_{\epsilon}^{\prime \prime}(x)$ is invertible and $\left.\left\|W_{\epsilon}^{\prime \prime}(x)^{-1}\right\| \leq c\left(\epsilon \delta^{2}\right)^{-1}\right\}$.

Theorem 2.1 (Existence). Let $W_{\epsilon}(x)$ satisfy conditions (A) and (B) with $m=3, \delta \ll 1$, and $\epsilon \ll \delta^{2}$. Suppose $W_{\mathrm{eff}, \epsilon}$ has a critical point at $z_{0} \in \Omega_{\epsilon \delta}$. Then for $\epsilon$ sufficiently small, there exist solutions of (1.6) and (1.7) of the form

$$
\psi_{\epsilon}(x)=e^{i \gamma(x)} \psi_{0}\left(x-z_{\epsilon}\right)+\xi_{\epsilon}(x), \quad A_{\epsilon}(x)=A_{0}\left(x-z_{\epsilon}\right)+\beta_{\epsilon}(x)+\nabla \gamma(x),
$$

where $z_{\epsilon}=z_{0}+O(\epsilon)$, the functions $\xi_{\epsilon}, \beta_{\epsilon}$ are $O(\epsilon)$ in $H^{2}\left(\mathbb{R}^{2} ; \mathbb{C}\right)$ and $H^{2}\left(\mathbb{R}^{2} ; \mathbb{R}^{2}\right)$, respectively, and $\gamma$ is an arbitrary $H^{1}\left(\mathbb{R}^{2} ; \mathbb{R}\right)$-function. 
Let $v=\left(\begin{array}{c}\psi \\ A\end{array}\right)$ and $v_{z \gamma}=\left(\begin{array}{c}\psi_{z \gamma} \\ A_{z \gamma}\end{array}\right)$, where

$$
\psi_{z \gamma}=e^{i\left(\gamma+z \cdot A_{z}\right)} \psi_{z} \quad A_{z \gamma}=A_{z}+\nabla\left(z \cdot A_{z}\right)+\nabla \gamma
$$

for $\gamma \in H^{1}\left(\mathbb{R}^{2} ; \mathbb{R}\right)$ and $z \in \mathbb{R}^{2}$. Here $\psi_{z}(x)=\psi_{0}(x-z)$ and $A_{z}=A_{0}(x-z)$.

We introduce the spaces $\mathbf{H}^{2}=H^{2}\left(\mathbb{R}^{2} ; \mathbb{C}\right) \oplus H^{2}\left(\mathbb{R}^{2} ; \mathbb{R}^{2}\right)$ and $\mathbf{L}^{2}=L^{2}\left(\mathbb{R}^{2} ; \mathbb{C}\right) \oplus$ $L^{2}\left(\mathbb{R}^{2} ; \mathbb{R}^{2}\right)$ equipped with the inner product

$$
\langle(\xi, B),(\eta, C)\rangle_{\mathbf{L}^{2}}=\int_{\mathbb{R}^{2}}\{\operatorname{Re}(\bar{\xi} \eta)+B \cdot C\} d x .
$$

Theorem 2.2 (Uniqueness). Let $W_{\epsilon}(x)$ satisfy conditions (A) and (B) with $m=3$, $\delta \ll 1$, and $\epsilon \ll \delta^{2}$. Assume for simplicity that $W_{\epsilon}$ is of the form $W_{\epsilon}(x)=\epsilon W(x)$. We have the following results:

(1) Suppose $z_{0} \in \Omega_{\epsilon \delta}$ is a bifurcation point, i.e., there exists a family of solutions $v_{\epsilon}$ of (1.6) and (1.7) with $v_{\epsilon} \rightarrow v_{z_{0} \gamma}$ in $\mathbf{H}^{2}$ for some $\gamma \in H^{1}\left(\mathbb{R}^{2} ; \mathbb{R}\right)$. Then $z_{0}$ is a critical point of $W_{\mathrm{eff}, \epsilon}$ for $\epsilon$ sufficiently small.

(2) Suppose $v_{\epsilon}, v_{\epsilon}^{\prime}$ are two families of solutions of (1.6) and (1.7) with $v_{\epsilon}, v_{\epsilon}^{\prime} \rightarrow v_{z_{0} \gamma}$ in $\mathbf{H}^{2}$ and $z_{0} \in \Omega_{\epsilon \delta}$. Then $v_{\epsilon}=v_{\epsilon}^{\prime}$ for $\epsilon$ sufficiently small.

Denote $v_{0}:=v_{z=0, \gamma=0}$. We will also need the affine space

$$
\mathbf{H}_{0}^{2}=v_{0}+\mathbf{H}^{2}=\left\{v_{0}+w \mid w \in \mathbf{H}^{2}\right\} .
$$

Theorem 2.3 (Reduced Energy). Under the assumptions of Theorem [2.1, for $\epsilon>0$ sufficiently small, there exists a constant $\delta_{0}>0$ and a $C^{3}$ function $\Phi_{\epsilon}: \mathbb{R}^{2} \rightarrow \mathbb{R}$ such that there is a one-to-one correspondence between the critical points of $\mathcal{E}_{\epsilon}$ in the tube $\left\{v \in \mathbf{H}_{0}^{2} \mid\left\|v-v_{z \gamma}\right\|_{\mathbf{H}^{2}} \leq \delta_{0}\right.$ for some $z \in \mathbb{R}^{2}$ and $\left.\gamma \in H^{1}\left(\mathbb{R}^{2} ; \mathbb{R}\right)\right\}$ and the critical points of $\Phi_{\epsilon}$ in $\mathbb{R}^{2}$.

The following proposition discusses the relationship between the critical points of $W_{\epsilon}$ and $W_{\text {eff }, \epsilon}$.

Proposition 2.1. Suppose our potential $W_{\epsilon}$ satisfies conditions (A) and (B) with $m=3$, $\delta \ll 1$. Then $W_{\epsilon}$ has a critical point (local minimum/maximum/saddle point) at $z^{\prime} \in$ $\Omega_{\epsilon \delta}$ if and only if $W_{\mathrm{eff}, \epsilon}$ has a critical point (local maximum/minimum/saddle point) at $z_{0} \in \Omega_{\epsilon \delta}$ with $\left|z^{\prime}-z_{0}\right| \leq c \delta$.

The proof of the following theorem will be given elsewhere [ST].

Theorem 2.4 (Type of a critical point). Suppose the assumptions of Theorem 2.1 are satisfied, and $W_{\epsilon}$ has a maximum/minimum or saddle point at $z^{\prime} \in \Omega_{\epsilon \delta}$. Then the corresponding solution $\left(\psi_{\epsilon}, A_{\epsilon}\right.$ ) (as guaranteed by Theorem 2.1 and Proposition 2.1) is a strict (modulo gauge symmetry) local minimizer/saddle point of $\mathcal{E}_{\epsilon}(\psi, A)$.

Theorems 2.1, 2.2, and 2.3 are proved in $\$ 3$, Proposition 2.1 will be proved in Subsection 6.4

Theorem 2.4 makes it possible to show that only vortices corresponding to maxima of the potential $W_{\epsilon}$ are orbitally stable (see [ST]).

Theorems 2.1, 2.2, and 2.4 give mathematical content to the physical picture of trapped vortices: out of a continuum of vortex solutions localized around arbitrary points of the physical space in the absence of an external potential, only few (modulo the gauge symmetry) solutions survive when such a potential is turned on; the latter solutions are localized near critical points of the potential, with only those localized near maxima being stable.

Theorem 2.3 introduces a reduced energy on $\mathbb{R}^{2}$ that gives a complete description of the vortex location and stability. 
Aftalion, Sandier, and Serfaty [ASaSe] proved a similar pinning result as in Theorem 2.1 in the $\lambda \rightarrow \infty$ regime, for applied external magnetic fields, bounded domains, and for maxima/minima of the potential. The result of [ASaSe] was extended to critical points of the external potential by Andre, Bauman, and Phillips $\mathrm{ABP}$. Chapman, Du, and Gunzburger [CDG] have done numerical work on pinning.

For reviews of the results on Ginzburg-Landau theory of superconductors, see [BFGLV, Gu1, JT, R, Riv1, Rub2. Some recent results on magnetic vortices can be found in [JMS]. Some of the issues touched upon in this paper were also addressed in GS2]. Earlier results related to trapping of solitons can be found in [FW, Oh1, Oh2, $\mathrm{ABC}$.

Remarks. (i) The restrictions on the parameters $\epsilon$ and $\delta$ in the statements above are not uniform in the parameter $\lambda$. Presumably, the restrictions imposed weaken as $\lambda$ increases; however, to prove this would require an additional estimate on the vortex profile (namely, a lower bound on $\int\left(1-f_{n}^{2}\right)$ needed in Lemma 6.2), which is not done in this paper.

(ii) We could have formulated the conditions on the potential $W_{\epsilon}$ entirely in terms of the effective potential $W_{\mathrm{eff}, \epsilon}$ so that no differentiability of $W_{\epsilon}$ would be required.

(iii) One can weaken our assumptions on $W_{\epsilon}$ considerably if one is interested in maxima and minima only.

\section{§3. Main steps yielding proof of Theorems 2.12 .3}

In this section, we describe the main steps yielding the proofs of Theorems 2.1, 2.2 and 2.3

Equations (1.6) and (1.7) are Euler-Lagrange equations for the Ginzburg-Landau energy functional with potential

$$
\mathcal{E}_{\epsilon}(\psi, A):=\frac{1}{2} \int_{\mathbb{R}^{2}}\left(\left|\nabla_{A} \psi\right|^{2}+(\nabla \times A)^{2}+\frac{\lambda}{4}\left(|\psi|^{2}-1\right)^{2}+W_{\epsilon}(x)\left(|\psi|^{2}-1\right)\right) d x
$$

defined on the space $\mathbf{H}_{0}^{2}$, i.e., the solutions of (1.6) and (1.7) are critical points of $\mathcal{E}_{\epsilon}$ : $\mathcal{E}_{\epsilon}^{\prime}(\psi, A)=0$. Here we note that $\mathcal{E}_{\epsilon}$ is $C^{\infty}$ if $W_{\epsilon} \in L^{2}$.

Let $v=(\psi, A) \in \mathbf{H}_{0}^{2}$; we introduce $F_{\epsilon}(v)=\mathcal{E}_{\epsilon}^{\prime}(v)$, defined as a map from $\mathbf{H}^{2}$ to $\mathbf{L}^{2}$. Explicitly,

$$
F_{\epsilon}(v):=\left(\begin{array}{c}
-\Delta_{A} \psi+\frac{\lambda}{2}\left(|\psi|^{2}-1\right) \psi+W_{\epsilon}(x) \psi \\
\nabla \times \nabla \times A+\operatorname{Im}\left(\bar{\psi} \nabla_{A} \psi\right)
\end{array}\right) .
$$

Thus, equations (1.6) and (1.7) can be written as $F_{\epsilon}(v)=0$.

Let $\pi_{z \gamma}$ denote the orthogonal projection onto the kernel $K_{z \gamma}$ of $F_{0}^{\prime}\left(v_{z \gamma}\right)$, and let $\pi_{z \gamma}^{\perp}:=1-\pi_{z \gamma}$. The operator $\pi_{z \gamma}^{\perp}$ projects onto the $\mathbf{L}^{2}$-orthogonal complement $K_{z \gamma}^{\perp}$ of $K_{z \gamma}$, i.e., $\pi_{z \gamma}^{\perp}: \mathbf{L}^{2} \rightarrow K_{z \gamma}^{\perp}$.

The proof of the existence of a solution of (1.6) and (1.7) relies on the following two steps.

Step 1. Liapunov-Schmidt reduction and solution in the orthogonal direction. We use Liapunov-Schmidt reduction to break the problem up into its tangential and orthogonal components to the infinite-dimensional manifold of approximate solutions:

$$
M:=\left\{v_{z \gamma} \mid z \in \mathbb{R}^{2}, \gamma \in H^{1}\left(\mathbb{R}^{2} ; \mathbb{R}\right)\right\} .
$$

First, we show that there exists a solution in the orthogonal direction. More precisely, we show that for all $z \in \mathbb{R}^{2}, \gamma \in H^{1}\left(\mathbb{R}^{2} ; \mathbb{R}\right)$ and $\epsilon$ sufficiently small, there exists a unique $w_{z \gamma \epsilon} \in K_{z \gamma}^{\perp}$ such that

$$
\pi_{z \gamma}^{\perp} F_{\epsilon}\left(v_{z \gamma}+w_{z \gamma \epsilon}\right)=0 .
$$


To do this, we show that the linearization of $F_{\epsilon}$ around a point $v_{z \gamma} \in M$ is invertible in the orthogonal direction. After deriving some estimates on the nonlinear term $N_{\epsilon}\left(v_{z \gamma}, w\right)$, we shall employ a fixed point argument to show the existence of a solution in the orthogonal direction.

Step 2. reduced problem and solution in the tangential direction. We solve the corresponding problem in the tangential direction. More precisely, we show that there exist $z_{\epsilon}$ such that

$$
\pi_{z_{\epsilon} \gamma} F_{\epsilon}\left(v_{z_{\epsilon} \gamma}+w_{z_{\epsilon} \gamma \epsilon}\right)=0 .
$$

To do this, we substitute the solution, $w_{z \gamma \epsilon}$, of (3.2) into the Ginzburg-Landau energy functional to get the reduced energy functional

$$
\Phi_{\epsilon}(z):=\mathcal{E}_{\epsilon}\left(v_{z \gamma}+w_{z \gamma \epsilon}\right)
$$

We show that (a) $\Phi_{\epsilon}(z)$ has a critical point at $z_{\epsilon}$ if and only if (3.3) has $z_{\epsilon}$ as a solution, and (b) if $W_{\text {eff, } \epsilon}(x)$ has a critical point at $z_{0} \in \Omega_{\epsilon \delta}$, then $\Phi_{\epsilon}(z)$ has a unique critical point at some $z_{\epsilon}=z_{0}+O(\epsilon)$ provided our potential $W_{\epsilon}$ satisfies (A) and (B) (with $m=3$ ) of Theorem $2.1 \delta \ll 1, \epsilon \ll \delta^{2}$.

Steps 1 and 2 imply $F_{\epsilon}\left(v_{z_{\epsilon} \gamma}+w_{z_{\epsilon} \gamma \epsilon}\right)=0$ and hence Theorem 2.1 follows. Step 1 will be carried out in 4 , and Steps $2(\mathrm{a})$ and $2(\mathrm{~b})$ will be carried out in $\$ \$ 5$ and 6 respectively. We give some essential details below.

Let $T_{v_{z \gamma}} M$ denote the tangent space to $M$ at $v_{z \gamma}$. Then

$$
T_{v_{z \gamma}} M=\left\{a \cdot \nabla_{z} v_{z \gamma}+\left\langle\sigma, \partial_{\gamma}\right\rangle v_{z \gamma} \mid a \in \mathbb{R}^{2}, \sigma \in H^{3}\left(\mathbb{R}^{2} ; \mathbb{R}\right)\right\} .
$$

Explicit expressions for the vectors $\partial_{z_{j}} v_{z \gamma}$ and $\partial_{\gamma(x)} v_{z \gamma}$ at $z=0$ and $\gamma=0$ (which suffice for our analysis; see the remark below) are given in equations (5.12) and (5.14). Here we only mention that due to our peculiar definition of the family $v_{z \gamma}$ (see equation (2.1)) the $\psi$-component of the $z_{j}$-derivative of $v_{z \gamma}$ can be expressed through the covariant derivative $\left(\partial_{j}-i A_{j}(x)\right) \psi_{0}(x)$. The latter fact implies that $\partial_{z_{j}} v_{z \gamma} \in H^{s}$ for any $s \geq 0$. Moreover, examining equations (5.12) and (5.14), we see that $T_{v_{z \gamma}} M \subset H^{2}\left(\mathbb{R}^{2} ; \mathbb{C}\right) \oplus H^{2}\left(\mathbb{R}^{2} ; \mathbb{R}^{2}\right)$.

Now, we state the critical fact we need in our analysis. To identify Theorem 3.1 with a result from GS1, we use the relation $F_{0}^{\prime}\left(v_{z \gamma}\right)=g_{z \gamma} F_{0}^{\prime}\left(v_{0}\right) g_{z \gamma}^{-1}$, where $g_{z \gamma}$ is defined by

$$
g_{z \gamma}\left(\begin{array}{l}
\psi \\
A
\end{array}\right):=\left(\begin{array}{c}
e^{i(\gamma+z \cdot A(\cdot-z))} \psi(\cdot-z) \\
A(\cdot-z)+\nabla(z \cdot A(\cdot-z))+\nabla \gamma
\end{array}\right)
$$

for $\gamma \in H^{1}\left(\mathbb{R}^{2} ; \mathbb{R}\right)$ and $z \in \mathbb{R}^{2}($ see (2.1) $)$.

Theorem 3.1 (GS1]). (i) $T_{v_{z \gamma}} M=\operatorname{Ker}\left[F_{0}^{\prime}\left(v_{z \gamma}\right)\right]$.

(ii) $\sigma_{c}\left(F_{0}^{\prime}\left(v_{z \gamma}\right)\right)=[\Sigma, \infty)$, where $\Sigma=\min (1, \lambda)$.

Corollary 3.1. $\left.F_{0}^{\prime}\left(v_{z \gamma}\right)\right|_{T_{v_{z \gamma}} M^{\perp}}$ is invertible.

Using Corollary 3.1, we shall prove the following main statement in Step 1. We denote by $B_{X}(z, r)$ the open ball of radius $r$ centered at $z$ in a Banach space $X$.

Theorem 3.2. Suppose $W_{\epsilon}(x)$ is $O(\epsilon)$ in $L^{2}$. Then there exist positive constants $\epsilon_{0}$ and $\delta_{0}$ such that for every $z \in \mathbb{R}^{2}, \gamma \in H^{1}\left(\mathbb{R}^{2} ; \mathbb{R}\right)$, and $0<\epsilon \leq \epsilon_{0}$, there is a unique element $w_{z \gamma \epsilon}$ in $B_{\mathbf{H}^{2}}\left(0, \delta_{0}\right) \cap K_{z \gamma}^{\perp}$ such that equation (3.2) is satisfied.

In addition, we have the following:

a) $\left\|w_{z \gamma \epsilon}\right\|_{\mathbf{H}^{2}} \leq D \epsilon$ with a constant $D=D\left(\|\psi\|_{\infty}, \kappa, \beta\right)>0, \kappa:=\sup _{\epsilon>0} \frac{\left\|W_{\epsilon}\right\|_{L^{2}}}{\epsilon}$, and $\beta$ is defined as in Lemma 4.1.

b) $w_{z \gamma \epsilon}$ is $C^{3}$ in $z$, and $\left\|\partial_{z_{i}}^{n} w_{z \gamma \epsilon}\right\|_{\mathbf{L}^{2}} \leq c \epsilon$ for $n=1,2,3$. 
Now, we shall state precisely part (a) of Step 2 in Theorem 3.3 below, but first we need some definitions.

We define a manifold diffeomorphic to the manifold $M$ of approximate solutions. By Theorem 3.2 for every $0<\epsilon \leq \epsilon_{0}, z \in \mathbb{R}^{2}$, and $\gamma \in H^{1}\left(\mathbb{R}^{2} ; \mathbb{R}\right)$ we have a unique $v_{z \gamma \epsilon}=v_{z \gamma}+w_{z \gamma \epsilon} \in \mathbf{H}_{0}^{2}$ with $\pi_{z \gamma}^{\perp} F_{\epsilon}\left(v_{z \gamma}+w_{z \gamma \epsilon}\right)=0$ and $w_{z \gamma \epsilon} \in \operatorname{Ran}\left(\pi_{z \gamma}^{\perp}\right)$. For $0<\epsilon \leq \epsilon_{0}$, we define the manifold $M_{\epsilon}$ by

$$
M_{\epsilon}=\left\{v_{z \gamma \epsilon} \in \mathbf{H}_{0}^{2} \mid v_{z \gamma \epsilon}=v_{z \gamma}+w_{z \gamma \epsilon}, \gamma \in H^{1}\left(\mathbb{R}^{2}, \mathbb{R}\right), z \in \mathbb{R}^{2}\right\} .
$$

Note that $M_{\epsilon}=M$ for $\epsilon=0$.

The effective energy $\Phi_{\epsilon}: \mathbb{R}^{2} \rightarrow \mathbb{R}$ is the energy functional $\mathcal{E}_{\epsilon}$ restricted to the manifold $M_{\epsilon}$. We have the following result.

Theorem 3.3. Assume the conditions of Theorem 3.2 and let $w_{z \gamma \epsilon}$ be a unique solution of (3.2) for given $z \in \mathbb{R}^{2}, \gamma \in H^{1}\left(\mathbb{R}^{2} ; \mathbb{R}\right)$, so that $\Phi_{\epsilon}(z)$ is well defined. Then for $0<\epsilon \leq \epsilon_{0}$, the reduced energy function $\Phi_{\epsilon}(z)$ has a critical point at $z=z_{\epsilon}$ if and only if equation (3.3) is satisfied.

Finally, in order to complete Step 2, we use part 1) of the following theorem.

Theorem 3.4. Suppose our potential $W_{\epsilon}$ satisfies conditions (A) and (B) in 1 2 with $m=3, \delta \ll 1$, and $\epsilon \ll \delta^{2}$. Then:

1) If $W_{\mathrm{eff}, \epsilon}$ has a critical point $z_{0} \in \Omega_{\epsilon \delta}$, then $\Phi_{\epsilon}$ has a unique critical point $z_{\epsilon} \in$ $B_{\mathbb{R}^{2}}\left(z_{0}, c \frac{1}{\delta}\right) \cap \Omega_{\epsilon \delta}$, and moreover $\left|z_{\epsilon}-z_{0}\right| \leq C \frac{\epsilon}{\delta^{2}}$.

2) If $\Phi_{\epsilon}$ has a critical point $z_{\epsilon} \in \Omega_{\epsilon \delta}$, then $W_{\mathrm{eff}, \epsilon}$ has a unique critical point $z_{0} \in$ $B_{\mathbb{R}^{2}}\left(z_{\epsilon}, c \frac{1}{\delta}\right) \cap \Omega_{\epsilon \delta}$, and moreover $\left|z_{0}-z_{\epsilon}\right| \leq C \frac{\epsilon}{\delta^{2}}$.

3) $W_{\mathrm{eff}, \epsilon}$ has a local minimum/maximum/saddle point at $z_{0}$ if and only if $\Phi_{\epsilon}$ has a local minimum/maximum/saddle point at $z_{\epsilon}$.

Theorems 3.2 3.3, and 3.4 are proved in $\S 44$, 5, and 6] respectively.

Now, we are ready to prove Theorems 2.12 .2 , and 2.3

Proof of Theorem 2.1. By Theorem 3.2, for all $z \in \mathbb{R}^{2}$ and $\gamma \in H^{1}\left(\mathbb{R}^{2} ; \mathbb{R}\right)$, there exists a unique $w_{z \gamma \epsilon} \in B_{\mathbf{H}^{2}}\left(0, \delta_{0}\right) \cap K_{z \gamma}^{\perp}$ such that $\pi_{z \gamma}^{\perp} F_{\epsilon}\left(v_{z \gamma}+w_{z \gamma \epsilon}\right)=0$. Therefore, $\Phi_{\epsilon}(z)=$ $\mathcal{E}_{\epsilon}\left(v_{z \gamma}+w_{z \gamma \epsilon}\right)$ is well defined. By Theorem 3.4 (part 1)), $\Phi_{\epsilon}$ has a critical point $z_{\epsilon} \in$ $B_{\mathbb{R}^{2}}\left(z_{0}, c \epsilon\right)$ for $\epsilon$ sufficiently small. Therefore, by Theorem 3.3, $\pi_{z_{\epsilon} \gamma} F_{\epsilon}\left(v_{z_{\epsilon} \gamma}+w_{z_{\epsilon} \gamma \epsilon}\right)=0$ and hence $F_{\epsilon}\left(v_{z_{\epsilon} \gamma}+w_{z_{\epsilon} \gamma \epsilon}\right)=0$. Moreover, $w_{z_{\epsilon} \gamma \epsilon}:=\left(\begin{array}{c}\xi_{\epsilon} \\ \beta_{\epsilon}\end{array}\right)=O(\epsilon)$ in $\mathbf{H}^{2}$ (by Theorem 3.2(a)). Denoting $\left(\begin{array}{c}\psi_{\epsilon} \\ A_{\epsilon}\end{array}\right):=v_{z_{\epsilon} \gamma}+w_{z_{\epsilon} \gamma \epsilon}$, we arrive at the statement of Theorem 2.1

Proof of Theorem 2.2. For part (1), let $z_{0} \in \Omega_{\epsilon \delta}$ be such that there exist solutions $v_{\epsilon_{k}}$ of $\mathcal{E}_{\epsilon_{k}}^{\prime}(v)=0$ satisfying $v_{\epsilon_{k}} \rightarrow v_{z_{0}}$ in $\mathbf{H}^{2}$. By Proposition 2 in Gu2] ("Splitting theorem"), there exists $k_{0}$ such that for all $k \geq k_{0}, v_{\epsilon_{k}}$ can be uniquely (modulo gauge) decomposed as $v_{\epsilon_{k}}=v_{z_{k} \gamma}+w_{k}$, where $z_{k} \rightarrow z_{0}$ and $w_{k} \perp T_{v_{z_{k}}} M$. Since $v_{\epsilon_{k}}$ is a solution of $\mathcal{E}_{\epsilon_{k}}^{\prime}(v)=0$, we have

$$
\pi_{z_{k}}^{\perp} F_{\epsilon_{k}}\left(v_{z_{k} \gamma}+w_{k}\right)=0 .
$$

Since $\left\|w_{k}\right\|_{\mathbf{H}^{2}} \rightarrow 0$ as $k \rightarrow \infty$, we can assume that $k_{0}$ is such that $\left\|w_{k}\right\|_{\mathbf{H}^{2}} \leq \delta_{0}$ for $k \geq k_{0}$, where $\delta_{0}$ is the same as in Theorem 3.2 Then, by Theorem 3.2, $w_{k}$ is a unique solution of (3.6) in $B_{\mathbf{H}^{2}}\left(0, \delta_{0}\right)$, and this solution satisfies $\left\|w_{k}\right\|_{\mathbf{H}^{2}} \leq D \epsilon_{k}$.

By Theorem 3.3 and (3.6), for all $k \geq k_{0}$ we have

$$
\nabla \Phi_{\epsilon_{k}}\left(z_{k}\right)=0 \text {. }
$$


Since $z_{0} \in \Omega_{\epsilon \delta}$ and $z_{k} \rightarrow z_{0}$, it follows that $z_{k} \in \Omega_{\epsilon \delta}$ for $k$ sufficiently large. Hence, by (3.7) and Theorem 3.4 (part 2)), for all $k \geq k_{0}$ we have

$$
\nabla W_{\mathrm{eff}, \epsilon_{k}}\left(z_{k}^{\prime}\right)=0 \quad \text { for some } z_{k}^{\prime} \in B_{\mathbb{R}^{2}}\left(z_{k}, \frac{c \epsilon_{k}}{\delta^{2}}\right) .
$$

Since $W_{\epsilon}=\epsilon W$, we have $W_{\text {eff }, \epsilon}=\epsilon W_{\text {eff }}$, where $W_{\text {eff }}(z)=\frac{1}{2} \int W(x)\left(|\psi(x-z)|^{2}-1\right) d x$. Since $W_{\text {eff }}$ is independent of $\epsilon$, the $z_{k}^{\prime}$ are independent of $k$ for all $k \geq k_{1}$ for some sufficiently large $k_{1}>k_{0}$. Since $z_{k}^{\prime} \rightarrow z_{0}$, we conclude that $z_{k}^{\prime}=z_{0}$ and, in particular, $\nabla W_{\text {eff }}\left(z_{0}\right)=0$. This implies that $W_{\text {eff, } \epsilon}$ has a critical point at $z_{0}\left(W_{\text {eff, } \epsilon}\right.$ has the same critical points for all $\epsilon$ ).

For part (2), suppose $v_{\epsilon}, v_{\epsilon}^{\prime}$ are solutions of $\mathcal{E}_{\epsilon}^{\prime}(v)=0$ with $v_{\epsilon}, v_{\epsilon}^{\prime} \rightarrow v_{z_{0}}$ in $\mathbf{H}^{2}$ and $z_{0} \in \Omega_{\epsilon \delta}$ (here and in the rest of the proof we omit the subscripts $\gamma$ ). Using Gu2 Proposition 2] once again, write $v_{\epsilon}^{\#}=v_{z_{\epsilon}^{\#}}+w_{z_{\epsilon}}$, where $v_{\epsilon}^{\#}$ is either $v_{\epsilon}$ or $v_{\epsilon}^{\prime}, z_{\epsilon}^{\#}$ is either $z_{\epsilon}$ or $z_{\epsilon}^{\prime}$, and $w_{z_{\epsilon}^{\#} \epsilon} \perp T_{z_{\epsilon}^{\#}} M$. As in the above argument in part (1), we have $w_{\epsilon z_{\epsilon}^{\#}}=O(\epsilon)$, $z_{\epsilon}^{\#} \rightarrow z_{0}$ as $\epsilon \rightarrow 0$, and $\nabla \Phi_{\epsilon}\left(z_{\epsilon}^{\#}\right)=0$. By the previous result, $\nabla W_{\mathrm{eff}, \epsilon}\left(z_{0}\right)=0$. Since $z_{0} \in \Omega_{\epsilon \delta}$, Theorem 3.4 (part 1)) implies that $\Phi_{\epsilon}$ has a unique critical point in $B_{\mathbb{R}^{2}}\left(z_{0}, c \frac{1}{\delta}\right)$. Since $z_{\epsilon}^{\#} \rightarrow z_{0}$ as $\epsilon \rightarrow 0$, for $\epsilon$ sufficiently small both $z_{\epsilon}$ and $z_{\epsilon}^{\prime}$ are in $B_{\mathbb{R}^{2}}\left(z_{0}, c \frac{1}{\delta}\right)$. Hence, $z_{\epsilon}=z_{\epsilon}^{\prime}$ for $\epsilon$ sufficiently small, i.e., $v_{\epsilon}=v_{\epsilon}^{\prime}$ for $\epsilon$ sufficiently small.

Proof of Theorem 2.3. Let a constant $\delta_{0}>0$ and a $C^{3}$ function $\Phi_{\epsilon}: \mathbb{R}^{2} \rightarrow \mathbb{R}$ be as in Theorems 3.2 and 3.3, respectively. By Theorem [3.2 for any $z, \gamma$ there exists a unique $w_{z \gamma \epsilon} \in B_{\mathbf{H}^{2}}\left(0, \delta_{0}\right) \cap K_{z \gamma}^{\perp}$ with $\pi_{z \gamma}^{\perp} F_{\epsilon}\left(v_{z \gamma}+w_{z \gamma \epsilon}\right)=0$. By Theorem 3.3 $\Phi_{\epsilon}$ has a critical point at $z_{\epsilon}$ if and only if $\pi_{z_{\epsilon} \gamma} F_{\epsilon}\left(v_{z_{\epsilon} \gamma}+w_{z_{\epsilon} \gamma \epsilon}\right)=0$. Hence, $\Phi_{\epsilon}$ has a critical point at $z=z_{\epsilon}$ if and only if $F_{\epsilon}\left(v_{z_{\epsilon} \gamma}+w_{z_{\epsilon} \gamma \epsilon}\right)=0$. Thus, $\Phi_{\epsilon}$ has a critical point in $\mathbb{R}^{2}$ if and only if $\mathcal{E}_{\epsilon}$ has a critical point in the tubular set specified in the theorem.

Remark. Some of the computations simplify if we transform the map $F_{\epsilon}$ "back" to a neighborhood of $v_{0}:=v_{00}$. This is done with the help of the transformation (3.5). Using this transformation, we write $v_{z \gamma}=g_{z \gamma} v_{0}$ and $F_{0}\left(g_{z \gamma} v\right)=g_{z \gamma} F_{0}(v)$, so that $F_{0}^{\prime}\left(g_{z \gamma} v\right)=g_{z \gamma} F_{0}^{\prime}(v) g_{z \gamma}^{-1}$. Next, we introduce the new map

$$
F_{\epsilon z}(v)=g_{z \gamma}^{-1} F_{\epsilon}\left(g_{z \gamma} v\right) \text {. }
$$

Observe the relations $F_{\epsilon z}^{\prime}(v)=g_{z \gamma}^{-1} F_{\epsilon}^{\prime}\left(g_{z \gamma} v\right) g_{z \gamma}$ and

$$
F_{\epsilon z}(v)=F_{0}(v)+\left(\begin{array}{c}
W_{\epsilon}(\cdot+z) \psi \\
0
\end{array}\right) .
$$

Now, instead of considering the equation $F_{\epsilon}(v)=0$ in a neighborhood of $v_{z \gamma}$, we consider the equation

$$
F_{\epsilon z}(v)=0
$$

in a neighborhood of the point $v_{0}$. In particular, instead of the equation

$$
\pi_{z \gamma}^{\perp} F_{\epsilon}\left(v_{z \gamma}+w\right)=0
$$

for $w \in\left(T_{v_{z \gamma}} M\right)^{\perp}$, we can consider the equation

$$
\pi_{0}^{\perp} F_{\epsilon z}\left(v_{0}+w\right)=0,
$$

where $\pi_{0}^{\perp}=1-\pi_{0}$ with $\pi_{0}=\pi_{00}$, for $w \in\left(T_{v_{0}} M\right)^{\perp}$. Similarly, instead of solving for $z$ the equation

$$
\pi_{z \gamma} F_{\epsilon}\left(v_{z \gamma}+w_{z \gamma \epsilon}\right)=0
$$

where $w_{z \gamma \epsilon} \in\left(T_{v_{z \gamma}} M\right)^{\perp}$ is the solution of (3.12), we can solve for $z$ the equation

$$
\pi_{0} F_{\epsilon z}\left(v_{0}+w_{z \epsilon}\right)=0,
$$


where $w_{z \epsilon} \in\left(T_{v_{0}} M\right)^{\perp}$ is the solution of (3.13). Observe that here

$$
w_{z \gamma \epsilon}=g_{z \gamma} w_{z \epsilon} \text {. }
$$

These are two equivalent approaches, and we use one or the other depending on its convenience in computations.

\section{§4. Solution in the orthogonal direction (proof of Theorem [3.2)}

In this section we prove that for $\epsilon$ sufficiently small, the equation $\pi_{z \gamma}^{\perp} F_{\epsilon}\left(v_{z \gamma}+w\right)=0$ for $w \in K_{z \gamma}^{\perp}$ has a unique solution. To this end we use an implicit function type argument. We begin with the following definitions. Let $v=v_{z \gamma}+w$, where $v_{z \gamma}=\left(\begin{array}{c}\psi_{z \gamma} \\ A_{z \gamma}\end{array}\right) \in M$, and $w=\left(\begin{array}{c}\xi \\ B\end{array}\right) \in \mathbf{H}^{2}$ with $w \perp T_{v_{z \gamma}} M$. We have the following Taylor expansion:

$$
F_{\epsilon}\left(v_{z \gamma}+w\right)=F_{\epsilon}\left(v_{z \gamma}\right)+F_{\epsilon}^{\prime}\left(v_{z \gamma}\right) w+N_{\epsilon}\left(v_{z \gamma}, w\right),
$$

where $N_{\epsilon}\left(v_{z \gamma}, w\right)$ is defined by this relation (the explicit form of $N_{\epsilon}\left(v_{z \gamma}, w\right)$ can be found in \$7). Define $L_{z \gamma \epsilon}=\left.\pi_{z \gamma}^{\perp} F_{\epsilon}^{\prime}\left(v_{z \gamma}\right)\right|_{K_{z \gamma}^{\perp} \cap \mathbf{H}^{2}}$.

To proceed, we need the following two lemmas the proofs of which are given in $₫ 7$

Lemma 4.1. There exist positive real numbers $\beta$ and $C_{1}$ independent of $\epsilon$, $z$, and $\gamma$ (see पf for the value of $C_{1}$ ) so that for $|\varepsilon| \ll C_{1}$ and $w \in K_{x y}^{\perp} \cap H^{2}$ we have

$$
\left\|L_{z \gamma \epsilon} w\right\|_{\mathbf{L}^{2}} \geq \beta\|w\|_{\mathbf{H}^{2}} .
$$

Lemma 4.2. There exist positive constants $C_{2}, C_{3}, C_{4}$ independent of $z, \gamma, \epsilon$ such that for all $w \in \mathbf{H}^{2}$ with $\|w\|_{\mathbf{H}^{2}} \leq C_{2}$,

$$
\left\|N_{\epsilon}\left(v_{z \gamma}, w\right)\right\|_{\mathbf{L}^{2}} \leq C_{3}\|w\|_{\mathbf{H}^{2}}^{2},
$$

and

$$
\left\|\partial_{w} N_{\epsilon}\left(v_{z \gamma}, w\right)\right\|_{\mathbf{H}^{2} \rightarrow \mathbf{L}^{2}} \leq C_{4}\|w\|_{\mathbf{H}^{2}} .
$$

Using the expansion (4.1) and abbreviating $\pi_{z \gamma}^{\perp} F_{\epsilon}\left(v_{z \gamma}\right)$ to $F_{z \gamma \epsilon}^{\perp}$ and $\pi_{z \gamma}^{\perp} N_{\epsilon}\left(v_{z \gamma}, w\right)$ to $N_{z \gamma \epsilon}^{\perp}(w)$, we rewrite equation (3.2) as a fixed point equation $w=S_{z \gamma \epsilon}(w)$ for the map $S_{z \gamma \epsilon}$ defined on $\mathbf{H}^{2}$ by

$$
S_{z \gamma \epsilon}(w)=-L_{z \gamma \epsilon}^{-1}\left[N_{z \gamma \epsilon}^{\perp}(w)+F_{z \gamma \epsilon}^{\perp}\right] .
$$

Let $\beta, C_{1}, C_{2}, C_{3}$ and $C_{4}$ be the constants occurring in Lemmas 4.1 and 4.2 and let $\kappa:=\sup _{\epsilon>0} \frac{1}{\epsilon}\left\|W_{\epsilon}\right\|_{L^{2}}$. Set $\delta_{0}=\min \left(C_{2}, \frac{\beta}{2 C_{3}}, \frac{\beta}{2 C_{4}}\right)$ and $\epsilon_{0}=\min \left(C_{1}, \frac{\delta_{0}}{2 \kappa} \beta\right)$. We shall show that, for $0<\epsilon \leq \epsilon_{0}$, the map $S_{z \gamma \epsilon}$ takes the ball $B_{\delta_{0}}^{\perp}=B_{\mathbf{H}^{2}}\left(0, \delta_{0}\right) \cap K_{z \gamma}^{\perp}$ continuously into itself. Let $w \in B_{\delta_{0}}^{\perp}$. Then by Lemmas 4.1 and 4.2. for $\epsilon<\epsilon_{0} \leq C_{1}$ and $\|w\| \leq \delta_{0} \leq C_{2}$, we have

$$
\left\|S_{z \gamma \epsilon}(w)\right\|_{\mathbf{H}^{2}} \leq \frac{1}{\beta}\left\|N_{z \gamma \epsilon}^{\perp}(w)+F_{z \gamma \epsilon}^{\perp}\right\|_{L^{2}} \leq \frac{1}{\beta}\left(C_{3}\|w\|_{\mathbf{H}^{2}}^{2}+\left\|F_{z \gamma \epsilon}^{\perp}\right\|_{\mathbf{L}^{2}}\right) .
$$

Since $F_{\epsilon}(v)=F_{0}(v)+\left(\begin{array}{cc}W_{\epsilon} & 0 \\ 0 & 0\end{array}\right) v$ and $F_{0}\left(v_{z \gamma}\right)=0$, we have $F_{\epsilon}\left(v_{z \gamma}\right)=\left(\begin{array}{c}W_{\epsilon} \psi_{z \gamma} \\ 0\end{array}\right)$; therefore (since $\left|\psi_{z \gamma}\right| \leq 1$ ),

$$
\left\|F_{z \gamma \epsilon}^{\perp}\right\|_{\mathbf{L}^{2}} \leq\left\|F_{\epsilon}\left(v_{z \gamma}\right)\right\|_{\mathbf{L}^{2}} \leq\left\|W_{\epsilon}\right\|_{L^{2}} \leq \kappa \epsilon .
$$

Combined with the preceding inequality, this gives

$$
\left\|S_{z \gamma \epsilon}(w)\right\|_{\mathbf{H}^{2}} \leq \frac{1}{\beta}\left(C_{3} \delta_{0}^{2}+\kappa \epsilon\right) \leq \delta_{0} .
$$

Therefore, $S_{z \gamma \epsilon}(w)$ is in $B_{\delta_{0}}^{\perp}$ too. 

that

In addition, for $w$ and $w^{\prime}$ in $B_{\delta_{0}}^{\perp}$, from (4.4) and the mean value theorem it follows

$$
\left\|N_{\epsilon}\left(v_{z \gamma}, w\right)-N_{\epsilon}\left(v_{z \gamma}, w^{\prime}\right)\right\|_{\mathbf{L}^{2}} \leq C_{4} \delta_{0}\left\|w-w^{\prime}\right\|_{\mathbf{H}^{2}} .
$$

Hence, (4.6) and our choice of $\delta_{0}$ imply

$$
\begin{aligned}
& \left\|S_{z \gamma \epsilon}(w)-S_{z \gamma \epsilon}\left(w^{\prime}\right)\right\|_{\mathbf{H}^{2}}=\left\|L_{z \gamma \epsilon}^{-1}\left(N_{z \gamma \epsilon}^{\perp}(w)-N_{z \gamma \epsilon}^{\perp}\left(w^{\prime}\right)\right)\right\|_{\mathbf{L}^{2}} \\
& \leq \frac{C_{4} \delta_{0}}{\beta}\left\|w-w^{\prime}\right\|_{\mathbf{H}^{2}} \leq \frac{1}{2}\left\|w-w^{\prime}\right\|_{\mathbf{H}^{2}} .
\end{aligned}
$$

Therefore, $S_{z \gamma \epsilon}$ is a contraction map, and so $S_{z \gamma \epsilon}$ has a unique fixed point $w_{z \gamma \epsilon}$ in $B_{\delta_{0}}^{\perp}$. By the definition of the map $S_{z \gamma \epsilon}$, this fixed point solves (3.2), which proves the first part of Theorem 3.2 .

For part a) of the second part of Theorem 3.2 we note that

$$
\left\|S_{z \gamma \epsilon}(0)\right\|_{\mathbf{H}^{2}}=\left\|L_{z \gamma \epsilon}^{-1} F_{z \gamma \epsilon}^{\perp}\right\|_{\mathbf{H}^{2}} \leq \beta^{-1}\left\|F_{\epsilon}\left(v_{z \gamma}\right)\right\|_{\mathbf{L}^{2}} .
$$

But for the fixed point $w_{z \gamma \epsilon}$ we have

$$
w_{z \gamma \epsilon}=S_{z \gamma \epsilon}\left(w_{z \gamma \epsilon}\right)=S_{z \gamma \epsilon}(0)+S_{z \gamma \epsilon}\left(w_{z \gamma \epsilon}\right)-S_{z \gamma \epsilon}(0) .
$$

Consequently,

$$
\begin{aligned}
\left\|w_{z \gamma \epsilon}\right\|_{\mathbf{H}^{2}} & \leq\left\|S_{z \gamma \epsilon}(0)\right\|_{\mathbf{H}^{2}}+\left\|S_{z \gamma \epsilon}\left(w_{z \gamma \epsilon}\right)-S_{z \gamma \epsilon}(0)\right\|_{\mathbf{H}^{2}} \\
& \leq \beta^{-1}\left\|F_{\epsilon}\left(v_{z \gamma}\right)\right\|_{\mathbf{L}^{2}}+\frac{1}{2}\left\|w_{z \gamma \epsilon}\right\|_{\mathbf{H}^{2}} .
\end{aligned}
$$

Since $\left\|F_{\epsilon}\left(v_{z \gamma}\right)\right\|_{\mathbf{L}^{2}} \leq \kappa \epsilon$ (see (4.5)) and $\beta$ is independent of $\epsilon$, the last inequality implies part a) with $D=2 \beta^{-1} \kappa$ :

$$
\left\|w_{z \gamma \epsilon}\right\|_{\mathbf{H}^{2}} \leq 2 \beta^{-1} \kappa \epsilon .
$$

To prove part b), we proceed in a standard way. Define $F^{\perp}: \mathbb{R}^{2} \times\left(\operatorname{Ran}\left(\pi_{z \gamma}\right)^{\perp} \cap \mathbf{H}^{2}\right) \rightarrow$ $\left(\operatorname{Ran}\left(\pi_{z \gamma}\right)^{\perp} \cap \mathbf{L}^{2}\right)$ by

$$
F^{\perp}(z, w)=\pi_{z \gamma}^{\perp} F_{\epsilon}\left(v_{z \gamma}+w\right)
$$

where we have suppressed the dependence of $F^{\perp}$ on $\epsilon$ and $\gamma$ for brevity. By part a), for $0<\epsilon \leq \epsilon_{0}$ and $\gamma \in H^{1}$ there exists $w=w(z) \in \operatorname{Ran}\left(\pi_{z \gamma}\right)^{\perp} \cap \mathbf{H}^{2}$ such that

$$
F^{\perp}(z, w(z))=0, \quad z \in \mathbb{R}^{2} .
$$

We shall show that $w(z)$ in $(4.9)$ is $C^{1}$ in $z$ (the $C^{2}$ and $C^{3}$ cases are similar). Fix $0<\epsilon \leq \epsilon_{0}$ and $\gamma \in H^{1}$. For any $z \in \mathbb{R}^{2}$ and sufficiently small $h \in \mathbb{R}^{2}$, we have by (4.9)

$$
F^{\perp}(z+h, w(z)+t)=0 \quad \text { where } t=w(z+h)-w(z) .
$$

Expanding the left-hand side of this equation around $(z, w(z))$ and using (4.9), we obtain

$$
\left\|\partial_{z} F^{\perp}(z, w(z)) h+\partial_{w} F^{\perp}(z, w(z)) t\right\|_{\mathbf{L}^{2}}=o\left(\|h\|_{\mathbb{R}^{2}}+\|t\|_{\mathbf{H}^{2}}\right) .
$$

Since $\partial_{w} F^{\perp}(z, w(z))$ is invertible, (4.11) implies

$$
\begin{aligned}
\| t+ & \left(\partial_{w} F^{\perp}(z, w(z))\right)^{-1} \partial_{z} F^{\perp}(z, w(z)) h \|_{\mathbf{H}^{2}} \\
& \leq\left\|\left(\partial_{w} F^{\perp}(z, w(z))\right)^{-1}\right\| \cdot o\left(\|h\|_{\mathbb{R}^{2}}+\|t\|_{\mathbf{H}^{2}}\right) .
\end{aligned}
$$

Now, suppose that $\|h\|_{\mathbb{R}^{2}}+\|t\|_{\mathbf{H}^{2}}$ is chosen so small that

$$
o\left(\|h\|_{\mathbb{R}^{2}}+\|t\|_{\mathbf{H}^{2}}\right)\left\|\partial_{w} F^{\perp}(z, w(z))^{-1}\right\|<1 / 2\left(\|h\|_{\mathbb{R}^{2}}+\|t\|_{\mathbf{H}^{2}}\right) .
$$

In addition, if $a:=2\left\|\partial_{w} F^{\perp}(z, w(z))^{-1} \cdot \partial_{z} F^{\perp}(z, w(z))\right\|+1$, then, using the triangle inequality on the left-hand side of (4.12), we deduce the estimate

$$
\|t\|_{\mathbf{H}^{2}}<a\|h\|_{\mathbb{R}^{2}}
$$


Equations (4.12) and (4.13) imply that

$$
\left\|t+\partial_{w} F^{\perp}(z, w(z))^{-1} \cdot \partial_{z} F^{\perp}(z, w(z)) h\right\|_{\mathbf{H}^{2}} \leq(a+1) o\left(\|h\|_{\mathbb{R}^{2}}\right),
$$

and by the definition of $t$ in (4.10), the above argument shows that $w(z)$ is $C^{1}$ in $z$, with $\partial_{z} w(z)$ given by

$$
\partial_{z} w(z)=-\partial_{w} F^{\perp}(z, w(z))^{-1} \partial_{z} F^{\perp}(z, w(z)) .
$$

Now we prove that $\left\|\partial_{z} w(z)\right\|_{\mathbf{H}^{2}} \leq c \epsilon$. Fix $z \in \mathbb{R}^{2}$ and write $w=w(z)$ for convenience. We estimate the right-hand side of (4.14). We have already shown before that $\left\|\partial_{w} F^{\perp}(z, w)^{-1}\right\| \leq C$. Hence, it suffices to check that $\left\|\partial_{z} F^{\perp}(z, w)\right\|=O(\epsilon)$ as $\epsilon \rightarrow 0$. By (4.8), we have

$$
\partial_{z} F^{\perp}(z, w)=\left(\partial_{z} \pi_{z \gamma}^{\perp}\right) F_{\epsilon}\left(v_{z \gamma}+w\right)+\pi_{z \gamma}^{\perp} \partial_{z} F_{\epsilon}\left(v_{z \gamma}+w\right) .
$$

By (5.8) and Lemmas [5.3 and [5.5] $\left\|\pi_{z \gamma}^{\perp}\right\| \leq C$. Similarly it can be shown that $\left\|\partial_{z} \pi_{z \gamma}^{\perp}\right\| \leq C$. By (4.1), we have

$$
\left\|F_{\epsilon}\left(v_{z \gamma}+w\right)\right\|_{\mathbf{L}^{2}} \leq\left\|W_{\epsilon}\right\|_{L^{2}}+C\|w\|_{\mathbf{H}^{2}}+C\|w\|_{\mathbf{H}^{2}}^{2}=O(\epsilon),
$$

because $\|w\|_{\mathbf{H}^{2}}=O(\epsilon)$ by (4.7). In the above, we have used equation (4.5) and the relation $W_{\epsilon}=O(\epsilon)$ in $L^{2}$ for the first term, the fact that $F_{\epsilon}$ is $C^{1}$ for the second term, and Lemma 4.2 for the last term. Now, recall that

$$
F_{\epsilon}^{\prime}\left(v_{z \gamma}\right) w=F_{0}^{\prime}\left(v_{z \gamma}\right) w+\left(\begin{array}{cc}
W_{\epsilon}(x) & 0 \\
0 & 0
\end{array}\right) w .
$$

By (4.17) and the fact that $F_{0}^{\prime}\left(v_{z \gamma}\right) \partial_{z} v_{z \gamma}=0$, we have

$$
\begin{aligned}
& \left\|\partial_{z} F_{\epsilon}\left(v_{z \gamma}+w\right)\right\|_{\mathbf{L}^{2}}=\left\|F_{\epsilon}^{\prime}\left(v_{z \gamma}+w\right) \partial_{z} v_{z \gamma}\right\|_{\mathbf{L}^{2}} \\
& \quad \leq\left\|\left(F_{\epsilon}^{\prime}\left(v_{z \gamma}+w\right)-F_{\epsilon}^{\prime}\left(v_{z \gamma}\right)\right) \partial_{z} v_{z \gamma}\right\|_{\mathbf{L}^{2}}+\left\|\left(\begin{array}{cc}
W_{\epsilon} & 0 \\
0 & 0
\end{array}\right) \partial_{z} v_{z \gamma}\right\|_{\mathbf{L}^{2}} \\
& \quad \leq C \cdot \max \left(\left\|\partial_{z} v_{z \gamma}\right\|_{\infty},\left\|\partial_{z}^{2} v_{z \gamma}\right\|_{\infty}\right)\|w\|_{\mathbf{H}^{2}}+\left\|W_{\epsilon}\right\|_{L^{2}}\left\|\partial_{z} v_{z \gamma}\right\|_{\infty} \\
& \quad=O(\epsilon),
\end{aligned}
$$

since $F_{\epsilon}$ is $C^{2}, W_{\epsilon}=O(\epsilon)$ in $L^{2}$, and $\left\|\partial_{z} v_{z \gamma}\right\|_{\infty},\left\|\partial_{z}^{2} v_{z \gamma}\right\|_{\infty}<\infty$ (by the explicit form of $\partial_{z} v_{z \gamma}$-see equation (2.1) and the line preceding it). Equations (4.15), (4.16), and (4.18) show that $\left\|\partial_{z} w_{z \gamma \epsilon}\right\|_{\mathbf{L}^{2}} \leq c \epsilon$ and we are done with the proof of the estimates in Theorem 3.2b) for $n=1$. The cases of $n=2,3$ are done similarly.

\section{§5. Critical point of Reduced Energy is equivalent to CRitical Point OF FULL ENERGY (PROOF OF THEOREM 3.3)}

In this section we show that the reduced energy $\Phi_{\epsilon}(z):=\mathcal{E}_{\epsilon}\left(v_{z \gamma \epsilon}\right)$ (we recall that $\left.v_{z \gamma \epsilon}:=v_{z \gamma}+w_{z \gamma \epsilon}\right)$ has a critical point at $z_{\epsilon}$ if and only if $\mathcal{E}_{\epsilon}^{\prime}\left(v_{z_{\epsilon} \gamma \epsilon}\right)=0$.

5.1. General Argument. Equation (3.2) implies that

$$
\mathcal{E}_{\epsilon}^{\prime}\left(v_{z \gamma \epsilon}\right) \in T_{v_{z \gamma}} M
$$

for any $z \in \mathbb{R}^{2}$. By the independence of the energy functional of gauge, we have

$$
0=\partial_{\gamma} \Phi_{\epsilon}(z)=\left\langle\partial_{\gamma} v_{z \gamma \epsilon}, \mathcal{E}_{\epsilon}^{\prime}\left(v_{z \gamma \epsilon}\right)\right\rangle .
$$

We claim that, given (5.1) and (5.2),

$$
\left.\partial_{z} \Phi_{\epsilon}(z)\right|_{z=z_{\epsilon}}=0 \Longleftrightarrow \mathcal{E}_{\epsilon}^{\prime}\left(\left.v_{z \gamma \epsilon}\right|_{z=z_{\epsilon}}\right)=0 .
$$


Note that statement (5.3) is equivalent to the statement in Theorem 3.3 . The $(\Leftarrow)$ part of statement (5.3) is trivial: if $\mathcal{E}_{\epsilon}^{\prime}\left(\left.v_{z \gamma \epsilon}\right|_{z=z_{\epsilon}}\right)=0$, then

$$
\left.\partial_{z} \Phi_{\epsilon}(z)\right|_{z=z_{\epsilon}}=\left.\left\langle\partial_{z} v_{z \gamma \epsilon}, \mathcal{E}_{\epsilon}^{\prime}\left(v_{z \gamma \epsilon}\right)\right\rangle\right|_{z=z_{\epsilon}}=\left\langle\left.\partial_{z} v_{z \gamma \epsilon}\right|_{z=z_{\epsilon}},\left.\mathcal{E}_{\epsilon}^{\prime}\left(v_{z \gamma \epsilon}\right)\right|_{z=z_{\epsilon}}\right\rangle=0 .
$$

Hence, it remains to prove the $(\Rightarrow)$ part of (5.3). First, we observe that the relation

$$
\left.\left\langle\partial_{z} v_{z \gamma \epsilon}, \mathcal{E}_{\epsilon}^{\prime}\left(v_{z \gamma \epsilon}\right)\right\rangle\right|_{z=z_{\epsilon}}=\left.\partial_{z} \Phi_{\epsilon}(z)\right|_{z=z_{\epsilon}}=0
$$

together with (5.2) implies

$$
\mathcal{E}_{\epsilon}^{\prime}\left(v_{z_{\epsilon} \gamma \epsilon}\right) \perp T_{v_{z_{\epsilon} \gamma \epsilon}} M_{\epsilon} .
$$

Thus, it remains to show that (5.1) and (5.5) imply $\left.\mathcal{E}_{\epsilon}^{\prime}\left(v_{z \gamma \epsilon}\right)\right|_{z=z_{\epsilon}}=0$. Let $f_{\epsilon}$ denote $\mathcal{E}_{\epsilon}^{\prime}\left(v_{z_{\epsilon} \gamma \epsilon}\right)$, and let

$$
\begin{aligned}
\pi & =L^{2} \text {-orthogonal projection onto } T_{v_{z_{\epsilon} \gamma}} M, \\
\pi_{\epsilon} & =L^{2} \text {-orthogonal projection onto } T_{v_{z_{\epsilon} \epsilon}} M_{\epsilon} .
\end{aligned}
$$

Then equations (5.1) and (5.5) can be written as

$$
\pi f_{\epsilon}=f_{\epsilon} \quad \text { and } \quad \pi_{\epsilon} f_{\epsilon}=0 .
$$

We want to show that $f_{\epsilon}=0$. But in view of (5.6),

$$
f_{\epsilon}=\pi f_{\epsilon}=\left(\pi-\pi_{\epsilon}\right) f_{\epsilon} .
$$

Now, by Proposition 5.1, we have

$$
\left\|f_{\epsilon}\right\| \leq\left\|\pi-\pi_{\epsilon}\right\|\left\|f_{\epsilon}\right\| \leq C \epsilon\left\|f_{\epsilon}\right\| .
$$

This implies that $f_{\epsilon}=0$, which completes the proof of the $(\Rightarrow)$ part of (5.3), modulo the proof of Proposition 5.1

Proposition 5.1. a) $\pi$ and $\pi_{\epsilon}$ are bounded, and b) $\left\|\pi-\pi_{\epsilon}\right\|=O(\epsilon)$.

Proof. The statement follows from (5.8), (5.9), and Lemmas 5.4 and 5.5 .

In the rest of this section, we only consider the case of $z=0$ and $\gamma=0$, to which the case of general $z$ and $\gamma$ can be reduced (see the remark at the end of 33 . We write $\psi$ and $A$ for $\psi_{0}$ and $A_{0}$.

5.2. The projections $\pi^{t}, \pi_{\epsilon}^{t}, \pi^{g}, \pi_{\epsilon}^{g}$. In this section, we find explicit expressions for the orthogonal projections $\pi$ and $\pi_{\epsilon}$.

Recall that $v_{z \gamma \epsilon}:=v_{z \gamma}+w_{z \gamma \epsilon}$. We set $T=\left(T_{1}, T_{2}\right)=\left.\nabla_{z} v_{z \gamma}\right|_{z=0, \gamma=0}$ and $G_{\delta}=$ $\left.\partial_{\gamma} v_{z \gamma}\right|_{z=0, \gamma=0}$, and $T^{\epsilon}=\left(T_{1}^{\epsilon}, T_{2}^{\epsilon}\right)=\left.\nabla_{z} v_{z \gamma \epsilon}\right|_{z=0, \gamma=0}$ and $G_{\delta}^{\epsilon}=\left.\partial_{\gamma} v_{z \gamma \epsilon}\right|_{z=0, \gamma=0}$. Since $T_{v} M=\left\{a \cdot T+\left\langle\sigma, G_{\delta}\right\rangle \mid a \in \mathbb{R}^{2}, \sigma \in H^{3}\left(\mathbb{R}^{2} ; \mathbb{R}\right)\right\}$ and $T_{v_{\epsilon}} M_{\epsilon}=\left\{a \cdot T^{\epsilon}+\left\langle\sigma, G_{\delta}^{\epsilon}\right\rangle \mid a \in\right.$ $\left.\mathbb{R}^{2}, \sigma \in H^{3}\left(\mathbb{R}^{2} ; \mathbb{R}\right)\right\}$, we have

$$
\begin{aligned}
\pi & =\pi^{t}+\pi^{g}, \\
\pi_{\epsilon} & =\pi_{\epsilon}^{t}+\pi_{\epsilon}^{g},
\end{aligned}
$$

where

$$
\begin{aligned}
\pi^{t} & =L^{2} \text {-orthogonal projection onto }\left\{a \cdot T \mid a \in \mathbb{R}^{2}\right\}, \\
\pi^{g} & =L^{2} \text {-orthogonal projection onto }\left\{\left\langle\sigma, G_{\delta}\right\rangle \mid \sigma \in H^{3}\left(\mathbb{R}^{2} ; \mathbb{R}\right)\right\}, \\
\pi_{\epsilon}^{t} & =L^{2} \text {-orthogonal projection onto }\left\{a \cdot T^{\epsilon} \mid a \in \mathbb{R}^{2}\right\}, \\
\pi_{\epsilon}^{g} & =L^{2} \text {-orthogonal projection onto }\left\{\left\langle\sigma, G_{\delta}^{\epsilon}\right\rangle \mid \sigma \in H^{3}\left(\mathbb{R}^{2} ; \mathbb{R}\right)\right\} .
\end{aligned}
$$

We recall that if $\left\{\phi_{i}\right\}$ is a basis for a subspace $V \subset X$, then the orthogonal projection $P_{V}$ onto $V$ is given by

$$
P_{V}=\sum\left|\phi_{i}\right\rangle\left(U^{-1}\right)_{i j}\left\langle\phi_{j}\right|
$$


where $(U)_{i j}=\left\langle\phi_{i} \mid \phi_{j}\right\rangle$ is the basis matrix, and where the labels $i$ and $j$ are allowed to vary through a continuous domain, in which case the sum in (5.11) should be understood as the corresponding integral.

Our goal is to compute the basis matrix for the basis $\left\{T_{j}, G_{\delta(x)}\right\}$. First, we compute $T_{j}$ and $G_{\delta_{x}}$. For the translational tangent vectors, we have explicitly

$$
T_{j}=\left(\begin{array}{c}
\left(\partial_{j}-i A_{j}\right) \psi \\
\partial_{j} A-\nabla A_{j}
\end{array}\right) \quad \text { for } j=1,2
$$

By using the formulas $\psi(x)=f_{1}(r) e^{i \theta}$ and $A(x)=a_{1}(r) \nabla \theta$ (for $x=(r, \theta)$ ), expression (5.12) can be further rewritten as

$$
T_{j}=\left(\begin{array}{c}
e^{i \theta}\left(\frac{x_{j}}{r} f_{1}^{\prime}+\left(1-a_{1}\right) \frac{x_{j}^{\perp}}{r^{2}} f_{1}\right) \\
\frac{a_{1}^{\prime}}{r} e_{j}^{\perp}
\end{array}\right)
$$

where $e_{1}^{\perp}=(0,1), e_{2}^{\perp}=(-1,0)$, and $x^{\perp}=\left(-x_{2}, x_{1}\right)$.

For the gauge tangent vectors, we have explicitly

$$
G_{\delta_{x}}=\left(\begin{array}{c}
i \delta_{x} \psi \\
-\nabla \delta_{x}
\end{array}\right) \quad G_{\delta_{x}}^{\epsilon}=\left(\begin{array}{c}
i \delta_{x} \psi^{\epsilon} \\
-\nabla \delta_{x}
\end{array}\right)
$$

where $\delta_{x}(y)=\delta(y-x)$.

Now, we compute the "basis matrices". Using (5.13) and the vanishing of the integrals of the type $\int x_{1} x_{2} \varphi(|x|) d x$, we obtain

$$
\left\langle T_{i}, T_{j}\right\rangle=\beta_{i} \delta_{i j} \quad \text { where } \beta_{i}=\pi \int_{\mathbb{R}^{2}}\left[\left(f_{1}^{\prime}(r)\right)^{2}+\frac{f_{1}^{2}(r)}{r^{2}}\left(1-a_{1}\right)^{2}+\frac{1}{\pi}\left(\frac{a_{1}^{\prime}(r)}{r}\right)^{2}\right] d x
$$

Since $T_{j}^{\epsilon}=T_{j}+\left.\partial_{z_{j}} w_{z \gamma \epsilon}\right|_{z=0, \gamma=0}$, we have

$$
\left\langle T_{i}^{\epsilon}, T_{j}^{\epsilon}\right\rangle=:\left(\beta^{\epsilon}\right)_{i j}=\beta_{i} \delta_{i j}+(\nu)_{i j}
$$

where $(\nu)_{i j}=\nu_{i j}=\left\langle T_{i}, \partial_{j} w\right\rangle+\left\langle\partial_{i} w, T_{j}\right\rangle+\left\langle\partial_{i} w, \partial_{j} w\right\rangle$, with $\partial_{j} w:=\left.\partial_{z_{j}} w_{z \gamma \epsilon}\right|_{z=0, \gamma=0}$. Note that $\|\nu\|=O(\epsilon)$ (since $\left\|\partial_{j} w\right\|_{\mathbf{L}^{2}}=O(\epsilon)$ by Theorem $3.2 \mathrm{~b}$ ) with $n=1$ ). Hence, the matrix $\beta^{\epsilon}$ is invertible (as a sum of an invertible matrix $\beta$ and a small matrix). In addition, using (5.14), we obtain

$$
\left\langle G_{\delta_{x}}, G_{\delta_{y}}\right\rangle=\left(-\Delta+|\psi|^{2}\right)(x, y), \quad\left\langle G_{\delta_{x}}^{\epsilon}, G_{\delta_{y}}^{\epsilon}\right\rangle=\left(-\Delta+\left|\psi^{\epsilon}\right|^{2}\right)(x, y), \quad\left\langle T_{j}, G_{\delta_{x}}\right\rangle=0 .
$$

Here we prove only the first of these relations. The other two can be proved in a similar way. Using (5.14), we write

$$
\begin{aligned}
\left\langle G_{\delta_{x}}, G_{\delta_{y}}\right\rangle & =\int \operatorname{Re}\left(\overline{i \delta_{x}(z) \psi(z)} i \delta_{y}(z) \psi(z)+\left(-\nabla_{z} \delta_{x}(z)\right) \cdot\left(-\nabla_{z} \delta_{y}(z)\right)\right) d z \\
& =\int \delta_{x}(z)\left(-\Delta+|\psi|^{2}\right) \delta_{y}(z) d z \\
& =\left\langle\delta_{x},\left(-\Delta+|\psi|^{2}\right) \delta_{y}\right\rangle .
\end{aligned}
$$

The expression on the right-hand side is the integral kernel of the operator $-\Delta+|\psi|^{2}$. 
Now, the projections introduced above can be expressed in terms of the bases $\left\{T_{j}, G_{\delta_{x}}\right\}$ and $\left\{T_{j}^{\epsilon}, G_{\delta_{x}}^{\epsilon}\right\}$. Using the equations above and (5.11), we have

$$
\begin{aligned}
\pi^{t} & =\sum_{i=1}^{2}\left|T_{i}\right\rangle \beta_{i}^{-1}\left\langle T_{i}\right| \\
\pi_{\epsilon}^{t} & =\sum_{i, j=1}^{2}\left|T_{i}^{\epsilon}\right\rangle\left(\left(\beta^{\epsilon}\right)^{-1}\right)_{i j}\left\langle T_{j}^{\epsilon}\right| \\
\pi^{g} & =\iint\left|G_{\delta_{x}}\right\rangle\left(-\Delta+|\psi|^{2}\right)^{-1}(x, y)\left\langle G_{\delta_{y}}\right| d x d y \\
\pi_{\epsilon}^{g} & =\iint\left|G_{\delta_{x}}^{\epsilon}\right\rangle\left(-\Delta+\left|\psi^{\epsilon}\right|^{2}\right)^{-1}(x, y)\left\langle G_{\delta_{y}}^{\epsilon}\right| d x d y .
\end{aligned}
$$

5.3. Boundedness of $\pi^{g}$. In this section, we prove the boundedness of the operator $\pi^{g}$. As a prelude to the calculations below, we note that there is a one-to-one correspondence between the operators $J$ on $H^{s}\left(\mathbb{R}^{2}\right)$ and the operators $\pi_{J}^{g}$ defined by

$$
\pi_{J}^{g}=\iint\left|G_{\delta_{x}}\right\rangle J(x, y)\left\langle G_{\delta_{y}}\right| d x d y,
$$

where $J(x, y)$ is the integral kernel of $J$. Moreover, this correspondence is linear, i.e., $\pi_{A}^{g}+\pi_{B}^{g}=\pi_{A+B}^{g}$. In our case (see (5.18)),

$$
\pi^{g}=\pi_{J}^{g} \quad \text { with } J=\left(-\Delta_{x}+|\psi(x)|^{2}\right)^{-1} .
$$

This can be seen from (5.17) and (5.18) $)$.

In the next lemma, we find explicitly how $\pi_{J}^{g}$ acts on vectors.

Lemma 5.1. If $f=\left(\begin{array}{c}\xi \\ B\end{array}\right)$, then, in the sense of distributions, we have

$$
\pi_{J}^{g} f=\left(\begin{array}{c}
i \psi J[\operatorname{Im}(\bar{\psi} \xi)+\nabla \cdot B] \\
\nabla(J[\operatorname{Im}(\bar{\psi} \xi)+\nabla \cdot B])
\end{array}\right)
$$

Proof. The computation

$$
\begin{aligned}
\left\langle G_{\delta_{y}} \mid f\right\rangle_{\mathbf{L}^{2}} & =\left\langle\left(\begin{array}{c}
i \delta_{y}(\cdot) \psi(\cdot) \\
\nabla \delta_{y}(\cdot)
\end{array}\right),\left(\begin{array}{c}
\xi(\cdot) \\
B(\cdot)
\end{array}\right)\right\rangle_{\mathbf{L}^{2}} \\
& =\operatorname{Re} \int\left(-i \delta_{y}(z) \overline{\psi(z)}\right) \xi(z) d z-\int \nabla_{z} \delta_{y}(z) \cdot B(z) d z \\
& =\operatorname{Im}(\overline{\psi(y)} \xi(y))+\nabla \cdot B(y)
\end{aligned}
$$

and (5.19) give (5.21).

We are ready to show the boundedness of $\pi_{J}$.

Lemma 5.2. Let $J: H^{s-1} \rightarrow H^{s+1}$ be a bounded operator. Then the operator $\pi_{J}^{g}$ : $\mathbf{H}^{s} \rightarrow \mathbf{H}^{s}$ is bounded, with

$$
\left\|\pi_{J}^{g}\right\|_{\mathbf{H}^{s} \rightarrow \mathbf{H}^{s}} \leq C\|J\|_{H^{s-1} \rightarrow H^{s+1}}
$$

The constant $C$ depends on $\left\|\partial_{x}^{|\alpha|} \psi_{0}\right\|_{\infty}$ for $|\alpha| \leq s$.

Proof. Estimate (15.22) follows from the explicit expression (5.21), the estimates $\partial_{x}^{|\alpha|} \psi_{0} \in$ $L^{\infty}$ for $|\alpha| \leq s$, and the boundedness of $J$ as an operator from $H^{s-1}$ to $H^{s+1}$. 
Lemma 5.3. For fixed $z \in \mathbb{R}^{2}$ and $\gamma \in H^{1}\left(\mathbb{R}^{2} ; \mathbb{R}\right)$, the operators $A:=-\Delta+|\psi|^{2}$ and $A_{\epsilon}:=-\Delta+\left|\psi^{\epsilon}\right|^{2}: H^{1} \rightarrow H^{-1}$ are invertible, and $J=A^{-1}$ and $J_{\epsilon}=A_{\epsilon}^{-1}$ satisfy all the conditions of Lemma 5.2 for $s=0$. Therefore, the operators $\pi^{g}=\pi_{A^{-1}}^{g}$ and $\pi_{\epsilon}^{g}=\pi_{A_{\epsilon}^{-1}}^{g}$ are bounded on $\mathbf{L}^{2}$ with the bound

$$
\left\|\pi_{\epsilon}^{g}\right\|_{\mathbf{L}^{2}} \leq C\left\|A_{\epsilon}^{-1}\right\|_{H^{-1} \rightarrow H^{1}}
$$

by Lemma 5.2, and (5.20) and similarly for $\pi^{g}$. Moreover, the quantities $\left\|A_{\epsilon}^{-1}\right\|_{H^{-1} \rightarrow H^{1}}$, $\epsilon \geq 0$, are bounded uniformly in $\epsilon$.

Proof. To show that the operator $A:=-\Delta+|\psi|^{2}: H^{2} \rightarrow L^{2}$ is invertible (with bounded inverse), we show that its spectrum is disjoint from zero. Clearly, $A$ is selfadjoint and positive. Hence, $\sigma(A) \subset[0, \infty)$. Now, since $|\psi|^{2} \rightarrow 1$ as $x \rightarrow \infty$, we have $\sigma_{\text {cont }}(A)=$ $[1, \infty)$ (see, e.g., $[\mathrm{HS}, \widehat{\mathrm{RS}}]$ ). Therefore, $A$ can have only discrete eigenvalues in $[0,1)$. Clearly, 0 is not an eigenvalue of $A$, whence $0 \notin \sigma(A)$. Therefore, $A: H^{2} \rightarrow L^{2}$ is invertible.

Now, to show that $\left(-\Delta+|\psi|^{2}\right)^{-1}: H^{-1} \rightarrow H^{1}$ is bounded, it suffices to check that the operator

$$
\left(-\Delta+|\psi|^{2}\right)^{-1}(-\Delta+1)^{1 / 2}: L^{2} \rightarrow H^{1} \quad \text { is bounded. }
$$

To prove (5.23), we write

$$
\begin{aligned}
(-\Delta+ & \left.|\psi|^{2}\right)^{-1}(-\Delta+1)^{1 / 2}=\left(-\Delta+|\psi|^{2}\right)^{-1}(-\Delta+1)^{-1 / 2}(-\Delta+1) \\
= & \underbrace{(-\Delta+1)^{-1 / 2}}_{H^{1} \leftarrow L^{2} \text { bounded }} \underbrace{\left(-\Delta+|\psi|^{2}\right)^{-1}(-\Delta+1)}_{L^{2} \leftarrow L^{2} \text { bounded }} \\
& +\underbrace{\left[\left(-\Delta+|\psi|^{2}\right)^{-1},(-\Delta+1)^{-1 / 2}\right](-\Delta+1)}_{B}
\end{aligned}
$$

since $\left(-\Delta+|\psi|^{2}\right)^{-1}(-\Delta+1)=1+\left(-\Delta+|\psi|^{2}\right)^{-1}\left(1-|\psi|^{2}\right)$, which is bounded from $L^{2}$ to $L^{2}$. Hence, all we must show is that $B: L^{2} \rightarrow H^{1}$ is bounded. Write

$$
B=-\underbrace{\left(-\Delta+|\psi|^{2}\right)^{-1}}_{H^{2} \leftarrow L^{2} \text { bounded }} \underbrace{\left[-\Delta+|\psi|^{2},(-\Delta+1)^{-1 / 2}\right]}_{L^{2} \leftarrow L^{2} \text { bounded }} \underbrace{\left(-\Delta+|\psi|^{2}\right)^{-1}(-\Delta+1)}_{L^{2} \leftarrow L^{2} \text { bounded }} .
$$

But we have $\left[-\Delta+|\psi|^{2},(-\Delta+1)^{-\frac{1}{2}}\right]=\left[|\psi|^{2},(-\Delta+1)^{-\frac{1}{2}}\right]$. Writing $(-\Delta+1)^{-\frac{1}{2}}=$ $c \int_{0}^{\infty} \frac{\omega^{-\frac{1}{2}}}{-\Delta+1+\omega} d w$, we see that $\left[|\psi|^{2},(-\Delta+1)^{-\frac{1}{2}}\right]$ is $\left(L^{2} \rightarrow L^{2}\right)$-bounded. Therefore, $B: L^{2} \rightarrow H^{1}$ is bounded, and we have proved (5.23).

The invertibility of the operators $A_{\epsilon}:=-\Delta+\left|\psi^{\epsilon}\right|^{2}: H^{2} \rightarrow L^{2}$ and the boundedness of $A_{\epsilon}^{-1}: H^{-1} \rightarrow H^{1}$ is proved similarly. Since $A_{\epsilon}$ is a small perturbation of $A$, one can show that the various norms of $A_{\epsilon}^{-1}$ considered here are bounded uniformly in $\epsilon$.

\subsection{Estimation of $\left\|\pi^{g}-\pi_{\epsilon}^{g}\right\|$.}

\section{Lemma 5.4.}

$$
\left\|\pi^{g}-\pi_{\epsilon}^{g}\right\|=O(\epsilon)
$$

Proof. By (5.18), we have

$$
\begin{aligned}
& \pi_{\epsilon}^{g}-\pi^{g}= \iint\left\{\left|G_{\delta_{x}}^{\epsilon}\right\rangle J_{\epsilon}(x, y)\left\langle G_{\delta_{y}}^{\epsilon}|-| G_{\delta_{x}}\right\rangle J_{\epsilon}(x, y)\left\langle G_{\delta_{y}}\right|\right\} d x d y \\
&=\iint\left\{\left|G_{\delta_{x}}^{\epsilon}-G_{\delta_{x}}\right\rangle J_{\epsilon}(x, y)\left\langle G_{\delta_{y}}^{\epsilon}\right|\right. \\
&\left.\quad+\left|G_{\delta_{x}}\right\rangle\left(J_{\epsilon}-J\right)(x, y)\left\langle G_{\delta_{y}}^{\epsilon}|+| G_{\delta_{x}}\right\rangle J(x, y)\left\langle G_{\delta_{y}}^{\epsilon}-G_{\delta_{y}}\right|\right\} d x d y,
\end{aligned}
$$


where $J(x, y)$ and $J_{\epsilon}(x, y)$ are the integral kernels of the operators $J:=\left(-\Delta+|\psi|^{2}\right)^{-1}$ and $J_{\epsilon}:=\left(-\Delta+\left|\psi^{\epsilon}\right|^{2}\right)^{-1}$. Since $G_{\delta_{x}}^{\epsilon}-G_{\delta_{x}}=\left(\begin{array}{c}i \delta_{x}\left(\psi^{\epsilon}-\psi\right) \\ 0\end{array}\right)$ and $J_{\epsilon}-J=J_{\epsilon}\left(|\psi|^{2}-\left|\psi^{\epsilon}\right|^{2}\right) J$, we have (by calculations in Lemma [5.1 and (5.24) for $f=\left(\begin{array}{l}\xi \\ B\end{array}\right) \in \mathbf{L}^{2}$

$$
\begin{aligned}
\left(\pi_{\epsilon}^{g}-\pi^{g}\right) f= & \left(\begin{array}{c}
i\left(\psi^{\epsilon}-\psi\right) J_{\epsilon}\left[\operatorname{Im}\left(\overline{\psi^{\epsilon}} \xi\right)+\nabla \cdot B\right] \\
0
\end{array}\right) \\
& +\left(\begin{array}{c}
i \psi\left(J_{\epsilon}\left(|\psi|^{2}-\left|\psi^{\epsilon}\right|^{2}\right) J\right)\left[\operatorname{Im}\left(\overline{\psi^{\epsilon}} \xi\right)+\nabla \cdot B\right] \\
\nabla\left(J_{\epsilon}\left(|\psi|^{2}-\left|\psi^{\epsilon}\right|^{2}\right) J\right)\left[\operatorname{Im}\left(\overline{\psi^{\epsilon}} \xi\right)+\nabla \cdot B\right]
\end{array}\right)+\left(\begin{array}{c}
i \psi J\left[\operatorname{Im}\left(\overline{\left(\psi^{\epsilon}-\psi\right)} \xi\right)\right] \\
\nabla J\left[\operatorname{Im}\left(\overline{\left(\psi^{\epsilon}-\psi\right)} \xi\right)\right]
\end{array}\right) .
\end{aligned}
$$

Now using the fact that $|\psi| \leq 1$ and $\left|\psi^{\epsilon}\right| \leq 1$, and using Theorem $3.2 \mathrm{a}$ ), we obtain $\left\|\left|\psi^{\epsilon}\right|^{2}-|\psi|^{2}\right\|_{\infty} \leq 2\left\|\psi^{\epsilon}-\psi\right\|_{\infty} \leq c \epsilon$. Since $J$ and $J_{\epsilon}$ are bounded from $H^{-1}$ to $H^{1}$ uniformly in $\epsilon$, we conclude that $\left\|\left(\pi_{\epsilon}^{g}-\pi^{g}\right) f\right\|_{\mathbf{L}^{2}} \leq c \epsilon\|f\|_{\mathbf{L}^{2}}$, which implies the statement of the lemma.

5.5. Estimation of $\left\|\pi_{\epsilon}^{t}-\pi^{t}\right\|$. Our goal in this section is to prove the following statement.

Lemma 5.5. The operators $\pi^{t}$ and $\pi_{\epsilon}^{t}$ are bounded and satisfy $\left\|\pi_{\epsilon}^{t}-\pi^{t}\right\|=O(\epsilon)$.

Proof. The operators $\pi^{t}$ and $\pi_{\epsilon}^{t}$ are simply $(2 \times 2)$-matrices, so the boundedness is trivial. Since $T_{i}^{\epsilon}=T_{i}+\partial_{i} w$, where, we recall, $\partial_{i} w=\left.\partial_{z_{i}} w_{z \gamma \epsilon}\right|_{z=0, \gamma=0}$, from (5.18) it follows that

$$
\begin{array}{r}
\pi_{\epsilon}^{t}-\pi^{t}=\sum_{i, j}\left|T_{i}\right\rangle\left(\beta^{-1}-\beta_{\epsilon}^{-1}\right)_{i j}\left\langle T_{j}|-| \partial_{i} w\right\rangle\left(\beta_{\epsilon}^{-1}\right)_{i j}\left\langle T_{j}\right| \\
-\left|T_{i}\right\rangle\left(\beta_{\epsilon}^{-1}\right)_{i j}\left\langle\partial_{j} w|-| \partial_{i} w\right\rangle\left(\beta_{\epsilon}^{-1}\right)_{i j}\left\langle\partial_{j} w\right| .
\end{array}
$$

Hence, we are reduced to showing that a) $\left\|\partial_{j} w\right\|_{\mathbf{L}^{2}}=O(\epsilon)$, b) $\left\|\beta_{\epsilon}^{-1}\right\|<C$ for $\epsilon$ sufficiently small, and c) $\left\|\beta_{\epsilon}^{-1}-\beta^{-1}\right\|=O(\epsilon)$. Theorem $3.2 \mathrm{~b}$ ) with $n=1$ takes care of a). For b), the invertibility of $\beta_{\epsilon}$ for $\epsilon<\epsilon_{0}$ small and the bound $\left\|\beta_{\epsilon}^{-1}\right\|<C$ follows from the representation $\beta_{\epsilon}=\beta+\nu$, where $\beta$ is independent of $\epsilon$ and is invertible, and $\|\nu\|=O(\epsilon)$ (see equation (5.16) and the paragraph after it).

Finally, c) follows from the relations

$$
\left\|\beta_{\epsilon}^{-1}-\beta^{-1}\right\|=\left\|\beta_{\epsilon}^{-1}\left(\beta-\beta_{\epsilon}\right) \beta^{-1}\right\| \leq\left\|\beta_{\epsilon}^{-1}\right\|\left\|\beta-\beta_{\epsilon}\right\|\left\|\beta^{-1}\right\|
$$

and $\left\|\beta-\beta_{\epsilon}\right\|=O(\epsilon)$ (see equation (15.16) and the paragraph after it).

\section{§6. Proof of Theorem 3.4}

Theorems 3.3 and 3.2 imply that the full energy $\mathcal{E}_{\epsilon}(v)$ has a critical point at $v_{\epsilon}=$ $v_{z_{\epsilon} \gamma}+w_{z_{\epsilon} \gamma \epsilon}$ if and only if the reduced energy $\Phi_{\epsilon}(z)=\mathcal{E}_{\epsilon}\left(v_{z \gamma}+w_{z \gamma \epsilon}\right)$ has a critical point at $z=z_{\epsilon}$. In this section we study the critical points of $\Phi_{\epsilon}(z)$.

Note that since $\mathcal{E}_{\epsilon}$ is $C^{\infty}$, and $v_{z \gamma}+w_{z \gamma \epsilon}$ is $C^{3}$ in $z$ as a vector-valued function from $\mathbb{R}^{2}$ to $\mathbf{H}^{2}$ (indeed, $z \rightarrow v_{z \gamma}=\left(\begin{array}{c}e^{i\left(\gamma+z \cdot A_{z}\right)} \psi_{z} \\ A_{z}+\nabla\left(z \cdot A_{z}\right)+\nabla \gamma\end{array}\right)$ is $C^{\infty}$ as can be deduced from the explicit representation, and $z \rightarrow w_{z \gamma \epsilon}$ is $C^{3}$ by Theorem $[3.2 \mathrm{~b})$, we see that $\Phi_{\epsilon}(z)$ is $C^{3}$.

6.1. Explicit Form of $\Phi_{\epsilon}(z)$. In this subsection we prove the following lemma.

Lemma 6.1. Suppose $W_{\epsilon}$ satisfies condition $(\mathrm{A})$. Then $\Phi_{\epsilon}(z)$ can be written as

$$
\Phi_{\epsilon}(z)=\mathcal{E}_{0}\left(\psi_{0}, A_{0}\right)+W_{\mathrm{eff}, \epsilon}(z)+R_{\epsilon}(z),
$$

where, we recall,

$$
W_{\mathrm{eff}, \epsilon}(z)=\frac{1}{2} \int_{\mathbb{R}^{2}} W_{\epsilon}(x)\left(\left|\psi_{0}(x-z)\right|^{2}-1\right) d x
$$


and

$$
\partial^{\alpha} R_{\epsilon}(z)=O\left(\epsilon^{2}\right), \quad 0 \leq|\alpha| \leq 3 .
$$

Proof. Equation (6.1) with

$$
R_{\epsilon}(z):=\mathcal{E}_{\epsilon}\left(v_{z \gamma}+w_{z \gamma \epsilon}\right)-\mathcal{E}_{\epsilon}\left(v_{z \gamma}\right)
$$

follows from the definition $\Phi_{\epsilon}(z):=\mathcal{E}_{\epsilon}\left(v_{z \gamma}+w_{z \gamma \epsilon}\right)$ and the relations

$$
\mathcal{E}_{\epsilon}\left(v_{z \gamma}\right)=\mathcal{E}_{0}\left(v_{z \gamma}\right)+\frac{1}{2} \int_{\mathbb{R}^{2}} W_{\epsilon}(x)\left(\left|\psi_{0}(x-z)\right|^{2}-1\right) d x
$$

and $\mathcal{E}_{0}\left(v_{z \gamma}\right)=\mathcal{E}_{0}\left(\psi_{0}, A_{0}\right)$ (by the translational and gauge invariance of the energy functional $\left.\mathcal{E}_{0}\right)$.

To prove (6.3) for $|\alpha|=0$, we notice that, since $\mathcal{E}_{0}^{\prime}\left(v_{z \gamma}\right)=0$, we have

$$
\mathcal{E}_{\epsilon}^{\prime}\left(v_{z \gamma}\right)=\left(\begin{array}{cc}
W_{\epsilon} & 0 \\
0 & 0
\end{array}\right) v_{z \gamma}=O(\epsilon)
$$

in $L^{2}$, and therefore

$$
R_{\epsilon}(z)=\langle\underbrace{\mathcal{E}_{\epsilon}^{\prime}\left(v_{z \gamma}\right)}_{O(\epsilon)}, \underbrace{w_{z \gamma \epsilon}}_{O(\epsilon)}\rangle+\underbrace{O\left(\|w\|^{2}\right)}_{O\left(\epsilon^{2}\right)} .
$$

For the proof of $(\underline{6.3})$ with $|\alpha|=1$, we use the fact that $\left\langle\mathcal{E}_{\epsilon}^{\prime \prime}\left(v_{z \gamma}\right) w_{z \gamma \epsilon}, \partial_{z} v_{z \gamma}\right\rangle=$ $\left\langle w_{z \gamma \epsilon}, \mathcal{E}_{\epsilon}^{\prime \prime}\left(v_{z \gamma}\right) \partial_{z} v_{z \gamma}\right\rangle=O\left(\epsilon^{2}\right)$, because $w_{z \gamma \epsilon}=O(\epsilon)$ by Theorem $\left.3.2 \mathrm{a}\right)$, and $\mathcal{E}_{\epsilon}^{\prime \prime}\left(v_{z \gamma}\right) \partial_{z} v_{z \gamma}$ $=O(\epsilon)$ by (4.17) and the identity $\mathcal{E}_{0}^{\prime \prime}\left(v_{z \gamma}\right) \partial_{z} v_{z \gamma}=0$. Also, we use the relations $\mathcal{E}_{\epsilon}^{\prime}\left(v_{z \gamma}+\right.$ $\left.w_{z \gamma \epsilon}\right)=O(\epsilon)$ (by (4.16) and $\partial_{z} w_{z \gamma \epsilon}=O(\epsilon)$ (by Theorem $3.2 \mathrm{~b}$ ) with $n=1$ ) in $L^{2}$. Then, by (6.4) and an expansion of $\mathcal{E}_{\epsilon}^{\prime}$ about $v_{z \gamma}$, we have

$$
\begin{aligned}
\partial_{z} R_{\epsilon}(z) & =\left\langle\mathcal{E}_{\epsilon}^{\prime}\left(v_{z \gamma}+w_{z \gamma \epsilon}\right), \partial_{z} v_{z \gamma}+\partial_{z} w_{z \gamma \epsilon}\right\rangle-\left\langle\mathcal{E}_{\epsilon}^{\prime}\left(v_{z \gamma}\right), \partial_{z} v_{z \gamma}\right\rangle \\
& =\underbrace{\left\langle\mathcal{E}_{\epsilon}^{\prime \prime}\left(v_{z \gamma}\right) w_{z \gamma \epsilon}, \partial_{z} v_{z \gamma}\right\rangle}_{O\left(\epsilon^{2}\right)}+\langle\underbrace{O\left(\|w\|^{2}\right)}_{O\left(\epsilon^{2}\right)}, \partial_{z} v_{z \gamma}\rangle+\langle\underbrace{\left\langle\mathcal{E}_{\epsilon}^{\prime}\left(v_{z \gamma}+w_{z \gamma \epsilon}\right)\right.}_{O(\epsilon)}, \underbrace{\partial_{z} w_{z \gamma \epsilon}}_{O(\epsilon)}\rangle .
\end{aligned}
$$

Using similar arguments as above and Theorem 3.2b) with $n=2,3$, we can prove (6.3) with $|\alpha|=2,3$.

6.2. The effective potential $W_{\mathrm{eff}, \epsilon}$. Here we prove estimates on $W_{\mathrm{eff}, \epsilon}(z)$. For future considerations, estimates in this subsection are more precise than needed. Namely, we track out the $\lambda$-dependence of the constants involved. Let $m_{\lambda}:=\min (\sqrt{\lambda}, 2)$ and $n_{\lambda}=$ $\int\left(1-\left|\psi_{0}(x)\right|^{2}\right) d x$. For a function $f: \mathbb{R}^{2} \rightarrow \mathbb{R}$ we denote by $f^{\prime}(z), f^{\prime \prime}(z)$ and $f^{\prime \prime \prime}(z)$, or $f^{(n)}(z), n=1,2,3$, the gradient, Hessian and third differential, respectively.

Lemma 6.2. Suppose $\delta \ll m_{\lambda}^{3} n_{\lambda}$ and $z \in \Omega_{\epsilon \delta}$. Then for $n=1,2,3$,

$$
W_{\mathrm{eff}, \epsilon}^{(n)}(z)=O\left(\epsilon \frac{\delta^{n}}{m_{\lambda}^{2}}\right) .
$$

Moreover, if $\left|W_{\mathrm{eff}, \epsilon}^{\prime}(z)\right| \ll \epsilon \delta n_{\lambda}$, then $W_{\mathrm{eff}, \epsilon}^{\prime \prime}(z)$ is invertible, with

$$
\left\|W_{\mathrm{eff}, \epsilon}^{\prime \prime}(z)^{-1}\right\| \leq c\left(\epsilon \delta^{2} n_{\lambda}\right)^{-1} .
$$

Proof. In the estimates below, we use the following bound proved in $\$ 9$

$$
\int|x|^{n}\left(1-\left|\psi_{0}(x)\right|^{2}\right) d x=O\left(m_{\lambda}^{-(n+2)}\right)
$$

Differentiating the equation $W_{\text {eff }, \epsilon}=\frac{1}{2} W_{\epsilon} *\left(\left|\psi_{0}\right|^{2}-1\right) n$ times and using condition (B) on $W_{\epsilon}$ and the bound (6.11), we arrive at (6.9). 
Now, we prove the second statement in Lemma 6.2, Expanding $W_{\epsilon}(x+z)$ in (6.2) about $z$ and using condition (B) on $W_{\epsilon}$ and estimate (6.11), we obtain $(6.12)$

$$
W_{\mathrm{eff}, \epsilon}^{\prime}(z)=\underbrace{W_{\epsilon}^{\prime}(z)}_{O(\epsilon \delta)} \underbrace{\int\left(\left|\psi_{0}(x)\right|^{2}-1\right) d x}_{O\left(\frac{1}{m_{\lambda}^{2}}\right)}+\int\{\underbrace{W_{\epsilon}^{\prime \prime}(z) x}_{0}+O\left(\epsilon \delta^{3} x^{2}\right)\}\left(\left|\psi_{0}(x)\right|^{2}-1\right) d x
$$

(the integral $\int x\left(\left|\psi_{0}\right|^{2}-1\right) d x$ vanishes since $\left|\psi_{0}(x)\right|^{2}$ is spherically symmetric), and

$$
W_{\mathrm{eff}, \epsilon}^{\prime \prime}(z)=-\underbrace{W_{\epsilon}^{\prime \prime}(z) \int\left(1-\left|\psi_{0}(x)\right|^{2}\right)}_{O\left(\epsilon \frac{\delta^{2}}{\left(m_{\lambda}\right)^{2}}\right)} d x-\int \underbrace{O\left(\epsilon \delta^{3} x\right)\left(1-\left|\psi_{0}(x)\right|^{2}\right)}_{O\left(\epsilon\left(\frac{\delta}{m_{\lambda}}\right)^{3}\right)} d x .
$$

If $\left|W_{\mathrm{eff}, \epsilon}^{\prime}(z)\right| \ll \epsilon \delta n_{\lambda}$, then (6.12) implies $\left|W_{\epsilon}^{\prime}(z)\right| \ll \epsilon \delta$ (since $\frac{\delta^{2}}{m_{\lambda}^{4}} \frac{1}{n_{\lambda}} \ll 1$ ). Since $z \in \Omega_{\epsilon \delta}$, $W_{\epsilon}^{\prime \prime}(z)$ is invertible and $\left\|W_{\epsilon}^{\prime \prime}(z)^{-1}\right\| \leq c\left(\epsilon \delta^{2}\right)^{-1}$. Since $\frac{\delta}{m_{\lambda}^{3}} \frac{1}{n_{\lambda}} \ll 1$, from 6.13) it follows that $W_{\text {eff }, \epsilon}^{\prime \prime}(z)$ is invertible with bound (6.10).

6.3. Critical points of $\Phi_{\epsilon}$. Now, we are ready to prove Theorem 3.4 The estimates below depend on $\lambda$, but this $\lambda$-guaranteed is not displayed explicitly. We start with part $1)$. Since $W_{\mathrm{eff}, \epsilon}^{\prime}\left(z_{0}\right)=0$, we have

$$
\Phi_{\epsilon}^{\prime}\left(z_{0}\right)=O\left(\epsilon^{2}\right)
$$

by (6.1) and (6.3).

Since $W_{\mathrm{eff}, \epsilon}^{\prime}\left(z_{0}\right)=0$ and $z_{0} \in \Omega_{\epsilon \delta}$, Lemma 6.2 shows that $W_{\mathrm{eff}, \epsilon}^{\prime \prime}\left(z_{0}\right)$ is invertible with the bound given in (6.10). Using this bound and the relations (6.1), (6.3), (6.10), and $\epsilon \ll \delta^{2}$, we see that $\Phi_{\epsilon}^{\prime \prime}\left(z_{0}\right)$ is invertible with the bound

$$
\left\|\Phi_{\epsilon}^{\prime \prime}\left(z_{0}\right)^{-1}\right\| \leq c^{\prime}\left(\epsilon \delta^{2}\right)^{-1} .
$$

By (6.9) with $n=3$, and by (6.1) and (6.3) with $|\alpha|=3$, we obtain

$$
\left\|\Phi_{\epsilon}^{\prime \prime \prime}\left(z_{0}\right)\right\|=O\left(\epsilon \delta^{3}+\varepsilon^{2}\right) .
$$

Hence, (6.14), (6.15), (6.16) and an implicit function theorem type argument (similar to that used in the proof of Proposition 2.1. see 88 imply that $\Phi_{\epsilon}$ has a unique critical point $z_{\epsilon} \in B_{\mathbb{R}^{2}}\left(z_{0}, C \frac{1}{\delta}\right)$ and $\left|z_{\epsilon}-z_{0}\right|=O\left(\frac{\epsilon}{\delta^{2}}\right)$.

To prove that $z_{\epsilon} \in \Omega_{\epsilon \delta}$, suppose $\left|W_{\epsilon}^{\prime}\left(z_{\epsilon}\right)\right| \ll \epsilon \delta$. We want to show that $W_{\epsilon}^{\prime \prime}\left(z_{\epsilon}\right)$ is invertible with the bound $\left\|W_{\epsilon}^{\prime \prime}\left(z_{\epsilon}\right)^{-1}\right\| \leq c\left(\epsilon \delta^{2}\right)^{-1}$. First, using the expansion

$$
W_{\epsilon}^{\prime}\left(z_{0}\right)=\underbrace{W_{\epsilon}^{\prime}\left(z_{\epsilon}\right)}_{o(\epsilon \delta)}+\underbrace{O\left(\epsilon \delta^{2}\left|z_{\epsilon}-z_{0}\right|\right)}_{O\left(\epsilon^{2}\right)}
$$

and the relation $\epsilon \ll \delta$, we obtain

$$
\left|W_{\epsilon}^{\prime}\left(z_{0}\right)\right| \ll \epsilon \delta .
$$

From (6.18) it follows that $W_{\epsilon}^{\prime \prime}\left(z_{0}\right)$ is invertible with the bound $\left\|W_{\epsilon}^{\prime \prime}\left(z_{0}\right)^{-1}\right\| \leq c\left(\epsilon \delta^{2}\right)^{-1}$ (since $z_{0} \in \Omega_{\epsilon \delta}$ ). This fact, the condition $\epsilon \ll \delta$, and the expansion

$$
W_{\epsilon}^{\prime \prime}\left(z_{\epsilon}\right)=\underbrace{W_{\epsilon}^{\prime \prime}\left(z_{0}\right)}_{O\left(\epsilon \delta^{2}\right)}+\underbrace{O\left(\epsilon \delta^{3}\left|z_{\epsilon}-z_{0}\right|\right)}_{O\left(\epsilon^{2} \delta\right)}
$$

(since $\left|z_{\epsilon}-z_{0}\right|=O\left(\frac{\epsilon}{\delta^{2}}\right)$ ) immediately imply that $z_{\epsilon} \in \Omega_{\epsilon \delta}$. This completes the proof of part 1) of Theorem 3.4

For part 2), note that the relations $\Phi_{\epsilon}^{\prime}\left(z_{\epsilon}\right)=0$, (6.1) and (6.3) imply

$$
W_{\mathrm{eff}, \epsilon}^{\prime}\left(z_{\epsilon}\right)=O\left(\epsilon^{2}\right) \text {. }
$$


Since $z_{\epsilon} \in \Omega_{\epsilon \delta}$ and $\epsilon \ll \delta$, Lemma 6.2 and (6.20) show that $W_{\mathrm{eff}, \epsilon}^{\prime \prime}\left(z_{\epsilon}\right)$ is invertible with the bound

$$
\left\|W_{\mathrm{eff}, \epsilon}^{\prime \prime}\left(z_{\epsilon}\right)^{-1}\right\| \leq c\left(\epsilon \delta^{2}\right)^{-1} .
$$

Hence, (6.20), 6.21), (6.9) with $n=3$ and an implicit function theorem type argument (similar to that used in the proof of Proposition 2.1; see $\$ 8$ imply that $W_{\mathrm{eff}, \epsilon}$ has a unique critical point $z_{0} \in B_{\mathbb{R}^{2}}\left(z_{\epsilon}, C \frac{1}{\delta}\right)$ and $\left|z_{0}-z_{\epsilon}\right|=O\left(\frac{\epsilon}{\delta^{2}}\right)$.

To show that $z_{0} \in \Omega_{\epsilon \delta}$, we use exactly the same arguments as those used in part 1) for the proof of the fact that $z_{\epsilon} \in \Omega_{\epsilon \delta}$. This completes the proof of part 2).

For part 3), first we prove the "only if" assertion. To fix the ideas, we assume that $W_{\mathrm{eff}, \epsilon}$ has a local minimum at $z_{0}$, i.e., $W_{\mathrm{eff}, \epsilon}^{\prime}\left(z_{0}\right)=0$ and $W_{\mathrm{eff}, \epsilon}^{\prime \prime}\left(z_{0}\right)>0$. Therefore, by Lemma 6.2 $W_{\mathrm{eff}, \epsilon}^{\prime \prime}\left(z_{0}\right) \geq c \epsilon \delta^{2}$. By part 1$), \Phi_{\epsilon}(z)$ has a unique critical point $z_{\epsilon}$ in $B_{\mathbb{R}^{2}}\left(z_{0}, C \frac{1}{\delta}\right) \cap \Omega_{\epsilon \delta}$. We want to show that $\Phi_{\epsilon}^{\prime \prime}\left(z_{\epsilon}\right)>0$. By (6.1) and (6.3), we have

$$
\Phi_{\epsilon}^{\prime \prime}\left(z_{\epsilon}\right)=W_{\mathrm{eff}, \epsilon}^{\prime \prime}\left(z_{\epsilon}\right)+O\left(\epsilon^{2}\right) .
$$

Hence, it suffices to check that $W_{\text {eff, } \epsilon}^{\prime \prime}\left(z_{\epsilon}\right) \geq c \epsilon \delta^{2}$ (since $\left.\epsilon \ll \delta^{2}\right)$. The latter follows from the expansion

$$
W_{\mathrm{eff}, \epsilon}^{\prime \prime}\left(z_{\epsilon}\right)=\underbrace{W_{\mathrm{eff}, \epsilon}^{\prime \prime}\left(z_{0}\right)}_{\geq c \epsilon \delta^{2}}+\underbrace{O\left(\epsilon \delta^{3}\left|z_{\epsilon}-z_{0}\right|\right)}_{O\left(\epsilon^{2} \delta\right)},
$$

and the condition $\epsilon \ll \delta$, and we are done with the "only if" statement of part 3).

For the "if" part, to fix the ideas we assume that $\Phi_{\epsilon}$ has a minimum at $z_{\epsilon}$, i.e., $\Phi_{\epsilon}^{\prime}\left(z_{\epsilon}\right)=0$ and $\Phi_{\epsilon}^{\prime \prime}\left(z_{\epsilon}\right)>0$. We want to show that $W_{\mathrm{eff}, \epsilon}^{\prime \prime}\left(z_{0}\right)>0$ for the critical point $z_{0}$ found in part 2) above. By (6.1), 6.3) and the assumption, we have

$$
W_{\mathrm{eff}, \epsilon}^{\prime \prime}\left(z_{\epsilon}\right)+O\left(\epsilon^{2}\right)=\Phi_{\epsilon}^{\prime \prime}\left(z_{\epsilon}\right)>0 .
$$

Since $\epsilon \ll \delta^{2}$ and $\left\|W_{\mathrm{eff}, \epsilon}^{\prime \prime}\left(z_{\epsilon}\right)^{-1}\right\| \leq c\left(\epsilon \delta^{2}\right)^{-1}$, (6.23) and spectral theory (see, e.g., [HS]) imply $W_{\mathrm{eff}, \epsilon}^{\prime \prime}\left(z_{\epsilon}\right) \geq c \epsilon \delta^{2}$. Hence, the result follows from the condition $\epsilon \ll \delta$ and the expansion

$$
W_{\mathrm{eff}, \epsilon}^{\prime \prime}\left(z_{0}\right)=\underbrace{W_{\mathrm{eff}, \epsilon}^{\prime \prime}\left(z_{\epsilon}\right)}_{\geq \epsilon \delta^{2}}+\underbrace{O\left(\epsilon \delta^{3}\left|z_{0}-z_{\epsilon}\right|\right)}_{O\left(\epsilon^{2} \delta\right)} .
$$

6.4. Proof of Proposition 2.1; relationship between $W_{\epsilon}$ and $W_{\mathrm{eff}, \epsilon}$. We first prove the "only if" part of the statement. Since $W_{\epsilon}^{\prime}\left(z^{\prime}\right)=0$, (6.12) implies

$$
W_{\text {eff }, \epsilon}^{\prime}\left(z^{\prime}\right)=O\left(\epsilon \delta^{3}\right) \text {. }
$$

Since $z^{\prime} \in \Omega_{\epsilon \delta}$ and $\delta \ll 1$, Lemma 6.2 and (6.24) show that $W_{\mathrm{eff}, \epsilon}^{\prime \prime}\left(z^{\prime}\right)$ is invertible with

$$
\left\|W_{\mathrm{eff}, \epsilon}^{\prime \prime}\left(z^{\prime}\right)^{-1}\right\| \leq c\left(\epsilon \delta^{2}\right)^{-1} .
$$

Hence, (6.24), 6.25), (6.9) with $n=3$ and an implicit function type argument (see 88 ) imply that $W_{\text {eff }, \epsilon}$ has a unique critical point at $z_{0} \in B_{\mathbb{R}^{2}}\left(z^{\prime}, c\right)$ and $\left|z_{0}-z^{\prime}\right|=O(\delta)$.

The fact that $z_{0} \in \Omega_{\epsilon \delta}$ is proved in the same way as the fact that $z_{\epsilon} \in \Omega_{\epsilon \delta}$ in the proof of part 1) of Theorem 3.4 (see the paragraph containing (6.17)-(6.19)).

For the "if" part of the statement, we note that the identity $W_{\mathrm{eff}, \epsilon}^{\prime}\left(z_{0}\right)=0$ and (6.12) imply

$$
W_{\epsilon}^{\prime}\left(z_{0}\right)=O\left(\epsilon \delta^{3}\right) .
$$

Since $\delta \ll 1$, (6.26) implies $\left|W_{\epsilon}^{\prime}\left(z_{0}\right)\right| \ll \epsilon \delta$, and since $z_{0} \in \Omega_{\epsilon \delta}$, we have

$$
\left\|W_{\epsilon}^{\prime \prime}\left(z_{0}\right)^{-1}\right\| \leq c\left(\epsilon \delta^{2}\right)^{-1} .
$$


By (6.26), (6.27) and an implicit function type argument (see (88), $W_{\epsilon}$ has a unique critical point at $z^{\prime} \in B_{\mathbb{R}^{2}}\left(z_{0}, c\right)$ and $\left|z^{\prime}-z_{0}\right|=O(\delta)$. Finally, the fact that $z^{\prime} \in \Omega_{\epsilon \delta}$ follows from the same argument as that mentioned above.

We note that (6.13) implies that $W_{\epsilon}$ has a local minimum/maximum if and only if $W_{\mathrm{eff}, \epsilon}$ has a local maximum/minimum.

\section{\$7. Proof of Lemmas 4.1 and 4.2}

In this section we drop the subscripts $z$ and $\gamma$ in $\psi$ and $A$ for brevity.

Proof of Lemma 4.1. Recall that, by [GS1, §3], for $w=\left(\begin{array}{c}\xi \\ B\end{array}\right)$,

$$
L w=\left(\begin{array}{c}
{\left[-\Delta_{A}+\frac{\lambda}{2}\left(2|\psi|^{2}-1\right)\right] \xi+\frac{\lambda}{2} \psi^{2} \bar{\xi}+i\left[2 \nabla_{A} \psi+\psi \nabla\right] \cdot B} \\
\operatorname{Im}\left(\left[\nabla_{A}^{-} \psi-\bar{\psi} \nabla_{A}\right] \xi\right)+\left(-\Delta+\nabla \nabla+|\psi|^{2}\right) \cdot B
\end{array}\right),
$$

where we use the notation $L=F_{0}^{\prime}\left(v_{z \gamma}\right)$. For $w \in K_{z \gamma}^{\perp}$, Theorem 3.1 yields

$$
\|L w\|_{\mathbf{L}^{2}} \geq \tau\|w\|_{\mathbf{L}^{2}}
$$

with $\tau=\inf (\sigma(L) \cap(0, \infty))$. By (7.1),

$$
\|L w\|_{\mathbf{L}^{2}} \geq(1-\alpha) \tau\|w\|_{\mathbf{L}^{2}}+\alpha\|L w\|_{\mathbf{L}^{2}} .
$$

Now, for $w=\left(\begin{array}{c}\xi \\ B\end{array}\right)$ we have

$$
\begin{aligned}
\|L w\|_{\mathbf{L}^{2}}^{2}= & \|\underbrace{\left[-\Delta_{A}+\frac{\lambda}{2}\left(2|\psi|^{2}-1\right)\right] \xi+\frac{\lambda}{2} \psi^{2} \bar{\xi}+i\left[2 \nabla_{A} \psi+\psi \nabla\right] \cdot B}_{(I)}\|_{L^{2}\left(\mathbb{R}^{2} ; \mathbb{C}\right)}^{2} \\
& +\|\underbrace{\operatorname{Im}\left(\left[\nabla_{A}^{-} \psi-\bar{\psi} \nabla_{A}\right] \xi\right)+\left(-\Delta+|\psi|^{2}+\nabla \nabla \cdot\right) B}_{(I I)}\|_{L^{2}\left(\mathbb{R}^{2} ; \mathbb{R}^{2}\right)}^{2}
\end{aligned}
$$

Let $w \in K_{z \gamma}^{\perp}$. Then $w$ is orthogonal to the gauge zero modes, i.e., $\operatorname{Im}(\bar{\psi} \xi)=\nabla \cdot B$. This and the inequalities

$$
\|\nabla \xi\|_{L^{2}} \leq \frac{1}{\sqrt{2} \delta_{1}}\|\xi\|_{L^{2}}+\frac{\delta_{1}}{\sqrt{2}}\|\Delta \xi\|_{L^{2}}
$$

for any $\delta_{1}>0$, and

$$
\|\nabla \nabla \cdot B\|_{L^{2}}=\|\nabla \operatorname{Im}(\bar{\psi} \xi)\|_{L^{2}} \leq \frac{C}{\delta_{2}}\|\xi\|_{L^{2}}+C \delta_{2}\|\Delta \xi\|_{L^{2}}
$$

for any $\delta_{2}>0$, imply that (assuming $\delta_{1}, \delta_{2} \leq 1$ )

$$
\|I\|_{L^{2}\left(\mathbb{R}^{2} ; \mathbb{C}\right)} \geq\left(1-C \delta_{1}\right)\|\Delta \xi\|_{L^{2}}-\frac{C}{\delta_{1}}\|\xi\|_{L^{2}}-C\|B\|_{L^{2}},
$$

and

$$
\|I I\|_{L^{2}\left(\mathbb{R}^{2} ; \mathbb{R}^{2}\right)} \geq\|\Delta B\|_{L^{2}\left(\mathbb{R}^{2} ; \mathbb{R}^{2}\right)}-C\left(\delta_{1}+\delta_{2}\right)\|\Delta \xi\|_{L^{2}}-\frac{C}{\delta_{1} \delta_{2}}\|\xi\|_{L^{2}}-C\|B\|_{L^{2}} .
$$

Choosing $\delta_{1}$ and $\delta_{2}$ sufficiently small, from (7.5) and (7.6) we deduce that

$$
\|L w\|_{\mathbf{L}^{2}} \geq \frac{1}{2}\left(\|\Delta B\|_{L^{2}\left(\mathbb{R}^{2} ; \mathbb{R}^{2}\right)}+\|\Delta \xi\|_{L^{2}}\right)-C\left(\|\xi\|_{L^{2}}+\|B\|_{L^{2}}\right)
$$

where $C>0$. This and (7.2) with $\alpha=\frac{\tau}{\tau+C}$ imply

$$
\|L w\|_{\mathbf{L}^{2}} \geq \beta^{\prime}\|w\|_{\mathbf{H}^{2}}
$$

where $\beta^{\prime}=\min \left\{\frac{\tau}{2}, \frac{\tau}{4(\tau+C)}\right\}$. 
To finish the proof of this lemma, we estimate, with the help of (4.17): $\left\|L_{z \gamma \epsilon} w\right\|_{\mathbf{L}^{2}} \geq$ $\|L w\|_{\mathbf{L}^{2}}-\left\|W_{\epsilon} \xi\right\|_{L^{2}} \geq\left(\beta^{\prime}-\left\|W_{\epsilon}\right\|_{L^{2}}\right)\|w\|_{\mathbf{H}^{2}}$. By the assumption on $W_{\epsilon}$, we have $\left\|W_{\epsilon}\right\|_{L^{2}} \leq$ $D \epsilon$. So, Lemma 4.1 follows with $\beta=\beta^{\prime}-\epsilon D$, and $C_{1}=\frac{\beta^{\prime}}{D}$.

Proof of Lemma 4.2. Recall the notation $v_{z \gamma}=\left(\begin{array}{c}\psi_{z \gamma} \\ A_{z \gamma}\end{array}\right)=\left(\begin{array}{c}\psi \\ A\end{array}\right)$, and let $w=\left(\begin{array}{l}\xi \\ B\end{array}\right) \in \mathbf{H}^{2}$. By (4.1), the remainder $N_{\epsilon}\left(v_{z \gamma}, w\right)$ can be computed explicitly as

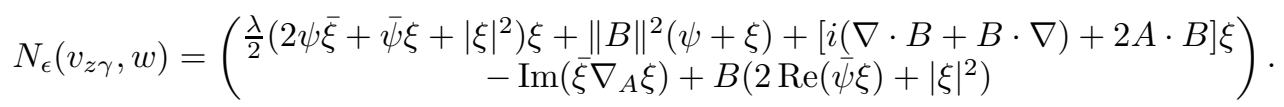

Using this formula, we compute for $\alpha=\left(\begin{array}{c}\eta \\ C\end{array}\right) \in \mathbf{H}^{2}$ :

$$
\begin{aligned}
\left(\partial_{w} N_{\epsilon}\left(v_{z \gamma}, w\right) \alpha\right)_{1}= & \lambda\left[\operatorname{Re}(\bar{\xi} \eta)(1+\xi)+\left(\xi+(1 / 2)|\xi|^{2}\right) \eta\right]+2 B \cdot C(\psi+\xi)+\|B\|^{2} \eta \\
& +[i(\nabla \cdot C+C \cdot \nabla)+2 A \cdot C] \xi+[i(\nabla \cdot B+B \cdot \nabla)+2 A \cdot B] \eta,
\end{aligned}
$$

and

$$
\left(\partial_{w} N_{\epsilon}\left(v_{z \gamma}, w\right) \alpha\right)_{2}=-\operatorname{Im}\left(\bar{\eta} \nabla_{A} \xi+\bar{\xi} \nabla_{A} \eta\right)+C\left(2 \operatorname{Re}(\bar{\psi} \xi)+|\xi|^{2}\right)+2 B \operatorname{Re}(\bar{\psi}+\xi) \eta .
$$

Now estimates (4.3) and (4.4) follow from the Sobolev embedding inequalities and estimates on $\psi$ and $A$.

\section{§8. An implicit FUnCTION TYPE ARGUMENT}

In this section we show that equations $(6.24), 6.25),(6.9)$ with $n=3$ imply that the function $W_{\text {eff, } \epsilon}$ has a unique critical point at $z_{0} \in B_{\mathbb{R}^{2}}\left(z^{\prime}, \alpha\right)$ for some $\alpha=O(1)$ and $\left|z_{0}-z^{\prime}\right|=O(\delta)$. For this, we use a standard implicit function type argument (see, e.g., $\mathrm{McO})$.

We begin with expanding $W_{\mathrm{eff}, \epsilon}^{\prime}(z)$ around $z^{\prime}$ to get

$$
W_{\mathrm{eff}, \epsilon}^{\prime}(z)=W_{\mathrm{eff}, \epsilon}^{\prime}\left(z^{\prime}\right)+W_{\mathrm{eff}, \epsilon}^{\prime \prime}\left(z^{\prime}\right) a+R_{\epsilon}(a),
$$

where $a=z-z^{\prime}$ and, by (6.9) with $n=3$,

$$
R_{\epsilon}(a)=O\left(\epsilon \delta^{3}|a|^{2}\right) .
$$

Using this expression and the fact that $W_{\mathrm{eff}, \epsilon}^{\prime \prime}\left(z^{\prime}\right)$ is invertible, we rewrite the equation $W_{\mathrm{eff}, \epsilon}^{\prime}(z)=0$ as $a=H(a)$, where

$$
H(a):=-W_{\mathrm{eff}, \epsilon}^{\prime \prime}\left(z^{\prime}\right)^{-1}\left(W_{\mathrm{eff}, \epsilon}^{\prime}\left(z^{\prime}\right)+R_{\epsilon}(a)\right) .
$$

Thus, $W_{\mathrm{eff}, \epsilon}^{\prime}\left(z_{0}\right)=0$ if and only if $H$ has a fixed point $z_{0}$.

To show that $H$ has a fixed point, we shall show that, for $\alpha=O(1)$ and $\delta$ sufficiently small,

1) $H$ maps $B_{\mathbb{R}^{2}}(0, \alpha) \rightarrow B_{\mathbb{R}^{2}}(0, \alpha)$;

2) $H$ is a contraction on $B_{\mathbb{R}^{2}}(0, \alpha)$.

We prove statement 1) first. By (6.24), (6.25), (8.1), and (8.2), we have

$$
\begin{aligned}
|H(a)| & \leq\left\|W_{\mathrm{eff}, \epsilon}^{\prime \prime}\left(z^{\prime}\right)^{-1}\right\|\left(\left|W_{\mathrm{eff}, \epsilon}^{\prime}\left(z^{\prime}\right)\right|+\left|R_{\epsilon}(a)\right|\right) \leq C\left(\epsilon \delta^{2}\right)^{-1}\left(\epsilon \delta^{3}+\epsilon \delta^{3}|a|^{2}\right) \\
& \leq \frac{C_{1}}{2} \delta+C_{2} \delta|a|^{2} .
\end{aligned}
$$

Now, choose $\alpha<\frac{1}{2 C_{2}}$ and $\delta<\frac{1}{C_{1}} \alpha$ (note that this is fulfilled because $\delta \ll 1$ by assumption). Then for $|a| \leq \alpha$, we have $|H(a)| \leq \alpha$, and we are done with 1$)$.

To prove 2), we use the fact that $W_{\mathrm{eff}, \epsilon}^{\prime}\left(z^{\prime}\right)+R_{\epsilon}(a)=W_{\mathrm{eff}, \epsilon}^{\prime}(z)-W_{\mathrm{eff}, \epsilon}^{\prime \prime}\left(z^{\prime}\right) a$. Then (8.2) implies that

$$
H^{\prime}(a)=-W_{\mathrm{eff}, \epsilon}^{\prime \prime}\left(z^{\prime}\right)^{-1}\left[W_{\mathrm{eff}, \epsilon}^{\prime \prime}(z)-W_{\mathrm{eff}, \epsilon}^{\prime \prime}\left(z^{\prime}\right)\right]
$$


for any $a \in B_{\mathbb{R}^{2}}(0, \alpha)$. Since $W_{\text {eff, } \epsilon}(z)$ is $C^{2}$ in $z$, we can choose $a$ to be sufficiently small so that $\left\|W_{\text {eff, } \epsilon}^{\prime \prime}(z)-W_{\text {eff, } \epsilon}^{\prime \prime}\left(z^{\prime}\right)\right\| \ll \frac{1}{2}\left\|W_{\text {eff, } \epsilon}^{\prime \prime}\left(z^{\prime}\right)^{-1}\right\|^{-1}$ for any $z \in B_{\mathbb{R}^{2}}\left(z^{\prime}, \alpha\right)$. Then $\left\|H^{\prime}(a)\right\| \leq 1 / 2$ for any $a \in B_{\mathbb{R}^{2}}(0, \alpha)$, and since $B_{\mathbb{R}^{2}}(0, \alpha)$ is a convex domain, it follows that $H$ is a contraction, and we are done with 2$)$.

Since the map $H$ is a contraction in $B_{\mathbb{R}^{2}}(0, \alpha)$, it has a unique fixed point there; therefore, there exists a unique $z_{0}$ in $B_{\mathbb{R}^{2}}\left(z^{\prime}, \alpha\right)$ such that $W_{\text {eff }, \epsilon}^{\prime}\left(z_{0}\right)=0$.

Moreover, for this fixed point $a$ we have

$$
|a|=|H(a)| \leq|H(0)|+|H(a)-H(0)| \leq C \delta+\frac{1}{2}|a|
$$

by (8.3) and because $H$ is a $1 / 2$-contraction. Thus, $\left|z-z_{0}\right|=|a|=O(\delta)$.

\section{$\S 9$. BOUNDS ON $a_{n}$ AND $f_{n}$}

In this section, we prove bounds on the vortex profiles $a_{n}$ and $f_{n}$ defined in (1.4). The index $n$ is fixed in what follows, and we write $f$ and $a$ for $f_{n}$ and $a_{n}$, respectively. In the proofs below, we use the following equations satisfied by $\xi:=1-f$ and $b:=1-a$, which follow from equations (1.1) and (1.2) and the definition (1.4):

$$
\begin{aligned}
{\left[-\Delta_{r}+\frac{n^{2} b^{2}}{r^{2}}+\frac{\lambda}{2} f(f+1)\right] \xi } & =\frac{n^{2} b^{2}}{r^{2}} \\
\left(-\Delta_{r}+\frac{2}{r} \frac{\partial}{\partial r}+f^{2}\right) b & =0
\end{aligned}
$$

where $\Delta_{r}=\frac{\partial^{2}}{\partial r^{2}}+\frac{1}{r} \frac{\partial}{\partial r}$.

We have the following exponential bounds on $\xi$ and $b$.

Theorem 9.1. Let $R=R(\epsilon)$ be such that

$$
f^{2}(r) \geq 2 \epsilon \quad \text { for } r=|x| \geq R,
$$

and let $\phi_{1}(r)=\int_{R}^{r} \sqrt{f^{2}(\rho)-\epsilon} d \rho$ and $\phi_{2}(r)=\min \left(\tilde{\phi}_{2}(r), 2 \phi_{1}(r)\right)$, where $\tilde{\phi}_{2}(r)=$ $\int_{R}^{r} \sqrt{\frac{\lambda}{2}[f(\rho)(f(\rho)+1)-\epsilon]} d \rho$. Then

$$
\left\|e^{\phi_{1}} b\right\|_{L^{2}} \leq C \epsilon^{-1} e^{R}\|b\|_{H^{1}}
$$

and

$$
\left\|e^{\phi_{2}} \xi\right\|_{L^{2}} \leq C \lambda^{-1} \epsilon^{-1}\left(e^{m_{\lambda} R}\|\xi\|_{H^{1}}+\epsilon C(R)\left\|e^{\phi_{1}} b\right\|_{L^{2}}^{2}\right),
$$

where $m_{\lambda}=\min (\sqrt{\lambda}, 2)$.

Proof. First, we obtain an exponential bound on $b$. We note that for any $v \in H^{2}\left(\mathbb{R}^{n} ; \mathbb{R}\right)$ and any $\phi_{1} \in C^{2}\left(\mathbb{R}^{n} ; \mathbb{R}\right)$ we have

$$
\operatorname{Re}\left\langle v, e^{\phi_{1}}(-\Delta) e^{-\phi_{1}} v\right\rangle=\left\langle v,\left(-\Delta-\left|\nabla \phi_{1}\right|^{2}\right) v\right\rangle .
$$

Observe that $\left\langle v, \frac{2}{r} \frac{\partial v}{\partial r}\right\rangle=2 \pi v(0)^{2}=0$ for any radially symmetric $H^{1}$-function $v$ with $v(0)=0$. Therefore, by $(9.3)$ and since $-\Delta_{r}$ is a positive operator, for any radially symmetric $H^{1}$-function $v$ with support in $\{r \geq R\}$ we have

$$
\left\langle v,\left(-\Delta_{r}+\frac{2}{r} \frac{\partial}{\partial r}+f^{2}-\left|\nabla \phi_{1}\right|^{2}\right) v\right\rangle \geq\left\langle v,\left(f^{2}-\left|\nabla \phi_{1}\right|^{2}\right) v\right\rangle \geq \epsilon\|v\|^{2} .
$$

Let $u=J e^{\phi_{1}} b$, and let $J$ be a $C^{\infty}$ function of $r$ such that $J=0$ if $r \leq R$ and $J=1$ if $r \geq R+1$ with $\left|\partial^{\alpha} J\right| \leq 2$ for $|\alpha| \leq 2$. Since $u$ is radially symmetric and $u(0)=0$, we 
can write

$$
\begin{aligned}
\epsilon\|u\|_{L^{2}}^{2} \leq & \left\langle u,\left(-\Delta_{r}+\frac{2}{r} \frac{\partial}{\partial r}+f^{2}-\left|\nabla \phi_{1}\right|^{2}\right) u\right\rangle \quad \text { by (9.7) } \\
& =\operatorname{Re}\left\langle u, e^{\phi_{1}}\left(-\Delta_{r}+\frac{2}{r} \frac{\partial}{\partial r}+f^{2}\right) e^{-\phi_{1}} u\right\rangle \quad \text { by (9.6) } \\
& =\operatorname{Re}\left\langle u, e^{\phi_{1}}\left[\left(-\Delta_{r}+\frac{2}{r} \frac{\partial}{\partial r}+f^{2}\right), J\right] b\right\rangle \quad \text { by } e^{-\phi_{1}} u=J b \text { and (9.2) } \\
& =\operatorname{Re}\left\langle u, e^{\phi_{1}}\left[-\Delta_{r}+\frac{2}{r} \frac{\partial}{\partial r}, J\right] b\right\rangle \\
\leq & \|u\|_{L^{2}}\left\|e^{\phi_{1}}\left[-\Delta_{r}+\frac{2}{r} \frac{\partial}{\partial r}, J\right] b\right\|_{L^{2}} .
\end{aligned}
$$

Since $\left[\Delta_{r}, J\right] b=2 \nabla J \cdot \nabla b+\Delta J \cdot b$ and $\left[\frac{2}{r} \frac{\partial}{\partial r}, J\right]=\frac{2}{r} \frac{\partial J}{\partial r}$, we have

$$
\begin{aligned}
\|u\|_{L^{2}} \leq & \epsilon^{-1}\left\{2\left\|e^{\phi_{1}} \nabla J\right\|_{\infty}\|\nabla b\|_{L^{2}}+\left\|e^{\phi_{1}} \nabla \cdot(\nabla J)\right\|_{\infty}\|b\|_{L^{2}}\right\} \\
& +\epsilon^{-1}\left\|e^{\phi_{1}} \frac{2}{r} \frac{\partial J}{\partial r}\right\|_{\infty}\|b\|_{L^{2}} .
\end{aligned}
$$

This gives

$$
\left\|J e^{\phi_{1}} b\right\|_{L^{2}} \leq C \epsilon^{-1} \sup _{x \in \operatorname{supp}(\nabla J)} e^{\phi_{1}}\|b\|_{H^{1}}
$$

To obtain an exponential bound $\xi$, we follow the same argument as above to prove (9.8). We use the fact that $-\Delta_{r}$ and $\frac{n^{2} b^{2}}{r^{2}}$ are positive operators and the inequality $\left|\nabla \phi_{2}\right|^{2} \leq \frac{\lambda}{2}[f(f+1)-\epsilon]$ to get

$$
\begin{aligned}
\left\langle v,\left(-\Delta_{r}+\frac{n^{2} b^{2}}{r^{2}}+\frac{\lambda}{2} f(f+1)-\left|\nabla \phi_{2}\right|^{2}\right) v\right\rangle & \geq\left\langle v,\left(\frac{\lambda}{2} f(f+1)-\left|\nabla \phi_{2}\right|^{2}\right) v\right\rangle \\
& \geq \frac{\lambda}{2} \epsilon\|v\|^{2}
\end{aligned}
$$

for any function $v$ with support in $\{r \geq R\}$. Let $u=J e^{\phi_{2}} \xi$, and let $J$ be the same function as defined in the proof of (9.4) above. We have

$$
\begin{array}{rlr}
\frac{\lambda}{2} \epsilon\|u\|_{L^{2}}^{2} & \leq\left\langle u,\left(-\Delta_{r}+\frac{n^{2} b^{2}}{r^{2}}+\frac{\lambda}{2} f(f+1)-\left|\nabla \phi_{2}\right|^{2}\right) u\right\rangle & \\
& =\operatorname{Re}\left\langle u, e^{\phi_{2}}\left(-\Delta_{r}+\frac{n^{2} b^{2}}{r^{2}}+\frac{\lambda}{2} f(f+1)\right) e^{-\phi_{2}} u\right\rangle & \text { by (9.6) } \\
& =\operatorname{Re}\left\langle u, e^{\phi_{2}}\left[-\Delta_{r}, J\right] \xi+e^{\phi_{2}} J \frac{n^{2} b^{2}}{r^{2}}\right\rangle & \text { by } e^{-\phi_{2}} u=J \xi \text { and (9.1) } \\
& \leq\|u\|_{L^{2}}\left\|e^{\phi_{2}}\left[-\Delta_{r}, J\right] \xi+e^{\phi_{2}} J \frac{n^{2} b^{2}}{r^{2}}\right\|_{L^{2}} .
\end{array}
$$

Therefore,

$$
\|u\|_{L^{2}} \leq 2 \lambda^{-1} \epsilon^{-1}\left(\left\|e^{\phi_{2}}\left[-\Delta_{r}, J\right] \xi\right\|_{L^{2}}+\left\|e^{\phi_{2}} J \frac{n^{2} b^{2}}{r^{2}}\right\|_{L^{2}}\right) .
$$

The first term can be estimated as in (9.8); this gives

$$
\left\|e^{\phi_{2}}\left[-\Delta_{r}, J\right] \xi\right\|_{L^{2}} \leq C \epsilon^{-1} \sup _{x \in \operatorname{supp}(\nabla J)} e^{\phi_{2}}\|\xi\|_{H^{1}} .
$$

For the second term, we use the fact that for any $\rho>0$ there is $d>0$ such that

$$
|b(x)| \leq d \int_{B_{\mathbb{R}^{2}}(x, \rho)}|b(y)| d y
$$


(see [CFKS, Theorem 2.4]). We have

$$
\begin{array}{rl}
\| e^{\phi_{2}} & J \frac{n^{2} b^{2}}{r^{2}} \|_{L^{2}}^{2} \\
& \leq\left(\frac{n}{R}\right)^{4} \int e^{2 \phi_{2}}|b|^{4} d x \\
& \leq\left(\frac{d n}{R}\right)^{4} \int d x e^{2 \phi_{2}}\left(\int_{B_{\mathbb{R}^{2}}(x, \rho)}|b(y)| d y\right)^{4} \\
& \leq\left(\frac{d n}{R}\right)^{4} \int d x e^{-\delta \phi_{2}}\left(\int_{B_{\mathbb{R}^{2}}(x, \rho)} e^{\left(\frac{1}{2}+\frac{\delta}{4}\right) \phi_{2}(y)+c \rho}|b(y)| d y\right)^{4} .
\end{array}
$$

Since $\phi_{2}(x) \leq \phi_{2}(\rho)+c \rho$ for $|x-y| \leq \rho$, the latter quantity is dominated by

$$
\left(\frac{d n}{R}\right)^{4} \int d x e^{-\delta c^{\prime}|x|}\left(\int_{B_{\mathbb{R}^{2}}(x, \rho)} d y\right)^{2}\left(\int_{B_{\mathbb{R}^{2}}(x, \rho)} e^{\left(1+\frac{\delta}{2}\right) \phi_{2}(y)+2 c \rho}|b(y)|^{2} d y\right)^{2} .
$$

Applying the Cauchy-Schwartz inequality and the estimate $\phi_{2}(x) \geq c^{\prime}|x|$, we find

$$
\left\|e^{\phi_{2}} J \frac{n^{2} b^{2}}{r^{2}}\right\|_{L^{2}}^{2} \leq\left(\frac{d n}{R}\right)^{4} \frac{1}{\delta c^{\prime}} \operatorname{vol}\left(B_{\mathbb{R}^{2}}(0, \rho)\right)^{2} e^{4 c \rho}\left\|e^{\left(\frac{1}{2}+\frac{\delta}{4}\right) \phi_{2}} b\right\|_{L^{2}}^{4} .
$$

Inequalities (9.9), (9.10), and (9.12) with $\rho=1$ imply the estimate

$$
\left\|J e^{\phi_{2}} \xi\right\|_{L^{2}} \leq C\left(\epsilon^{-1} \sup _{x \in \operatorname{supp}(\nabla J)} e^{\phi_{2}}\|\xi\|_{H^{1}}+\delta^{-1} R^{-4}\left\|e^{\left(\frac{1}{2}+\frac{\delta}{4}\right) \phi_{2}} b\right\|_{L^{2}}^{4}\right) .
$$

Now, by increasing constants, we can replace $\phi_{1}$ and $\phi_{2}$ on the right-hand sides of (9.8) and (9.13) by $r$ and $m_{\lambda} r$, respectively. Here, $m_{\lambda}=\min (\sqrt{\lambda}, 2)$. Since $r \leq R+1$ on $\operatorname{supp}(1-J)$, we have $\left\|e^{\phi_{1}} b\right\|^{2} \leq\left\|J e^{\phi_{1}} b\right\|^{2}+e^{2(R+1)}\|(1-J) b\|^{2}$. Combined with (9.8), this yields (9.4). Inequality (9.5) is obtained similarly.

Picking now $R_{\text {new }}=R_{\text {new }}(\epsilon)$ so that $f^{2}(r) \geq 1-\epsilon$ for $r \geq R_{\text {new }}$, we obtain

$$
\phi_{1}(r) \geq(1-2 \epsilon)\left(r-R_{\text {new }}\right)
$$

and

$$
\phi_{2}(r) \geq \min (\sqrt{\lambda}, 2)(1-2 \epsilon)\left(r-R_{\text {new }}\right) \quad \text { for } r \geq R_{\text {new }} .
$$

Using (9.14) in (9.4) and (9.5) in Theorem 9.1 and using (9.11) once again, we arrive at the following statement.

Corollary 9.1. Let $m_{\lambda}=\min (\sqrt{\lambda}, 2)$, and let $R \equiv R_{\text {new }}$ be as in 9.14). Then

$$
1-\left|f_{n}\right| \leq c(R) e^{-m_{\lambda}|x|} \quad \text { and } \quad 1-\left|a_{n}\right| \leq c(R) e^{-|x|} .
$$

\section{REFERENCES}

[ABC] A. Ambrosetti, M. Badiale, and S. Cingolani, Semiclassical states of nonlinear Schrödinger equations, Arch. Rational Mech. Anal. 140 (1997), 285-300. MR.1486895 (98k:35172)

[ABP $] \quad$ N. Andre, P. Bauman, and D. Phillips, Vortex pinning with bounded fields for the GinzburgLandau equation, Ann. Inst. H. Poincaré Anal. Non Linéaire 20 (2003), no. 4, 705-729. MR.1981405 (2004k:58024)

[ASaSe] A. Aftalion, E. Sandier, and S. Serfaty, Pinning phenomena in the Ginzburg-Landau model of superconductivity, J. Math. Pure Appl. (9) 80 (2001), no. 3, 339-372. MR1826348 (2002i:35018) 
[BC] M. S. Berger and Y. Y. Chen, Symmetric vortices for the Ginzburg-Landau equations of superconductivity, and the nonlinear desingularization phenomenon, J. Funct. Anal. 82 (1989), 259-295. MR0987294 (90f:58026)

[BFGLV] G. Blatter, M. V. Feigel'man, V. B. Geshkenbein et al., Vortices in high temperature superconductors, Rev. Modern Phys. 66 (1994), 112.5.

[CR1] S. J. Chapman and G. Richardson, Motion of vortices in type-II superconductors, SIAM J. Appl. Math. 55 (1995), 1275-1296. MR1349310 (97d:82086)

[CR2] - Vortex pinning by inhomogeneities in type-II superconductors, Phys. D 108 (1997), 397-407. MR:1474691 (98k:82201)

[CR3] Motion and homogenization of vortices in anisotropic type II superconductors, SIAM J. Appl. Math. 58 (1998), 587-606 (electronic). MR1617626|(99c:82073)

[CDG] S. J. Chapman, Q. Du, and M. D. Gunzburger, A Ginzburg-Landau type model of superconducting/normal junctions including Josephson junctions, European J. Appl. Math. 6 (1995), 97-114. MR1331493 (96c:82069)

[CFKS] H. L. Cycon, R. G. Froese, W. Kirsch, and B. Simon, Schrödinger operators with application to quantum mechanics and global geometry, Springer-Verlag, Berlin, 1987. MR0883643 (88g:35003)

[DG] Q. Du and M. D. Gunzburger, A model for superconducting thin films having variable thickness, Phys. D 69 (1993), 215-231. MR1251263 (94j:82087)

[DGP] Q. Du, M. D. Gunzburger, and J. S. Peterson, Computational simulations of type II superconductivity including pinning phenomena, Phys. Rev. B 51 (1995), no. 22.

[FW] A. Floer and A. Weinstein, Nonspreading wave packets for the cubic Schrödinger equation with a bounded potential, J. Funct. Anal. 69 (1986), 397-408. MR0867665 (88d:35169)

[GT] D. Gilbarg and N. S. Trudinger, Elliptic partial differential equations of second order, 2nd. ed., Springer-Verlag, Berlin, 1998. MR0737190 (86c:35035)

[Gu1] S. Gustafson, Some mathematical problems in the Ginzburg-Landau theory of superconductivity, Ph. D. Thesis, Univ. Toronto, 1999.

[Gu2] - Dynamic stability of magnetic vortices, Nonlinearity 15 (2002), 1717-1728. MR 1925436 (2003i:35251)

[GS1] S. Gustafson and I. M. Sigal, The stability of magnetic vortices, Comm. Math. Phys. 212 (2000), no. 2, 257-275. MR.1772246 (2002h:58018)

[GS2] Dynamics of magnetic vortices, Preprint, 2003.

[HS] P. D. Hislop and I. M. Sigal, Introduction to spectral theory, Appl. Math. Sci., vol. 113, Springer-Verlag, New York, 1996. MR1361167 (98h:47003)

[JMS] R. Jerrard, A. Montero, and P. Sternberg, Local minimizers of the Ginzburg-Landau energy with magnetic field in three dimensions, Preprint, 2003.

[JT] A. Jaffe and C. Taubes, Vortices and monopoles. Structure of static gauge theories, Progr. in Phys., vol. 2, Bikhäuser, Boston, MA, 1980. MR0614447 (82m:81051)

[McO] R. C. McOwen, Partial differential equations. Methods and applications, Prentice-Hall, Upper Saddle River, NJ, 1996.

[Oh1] Y.-G. Oh, Existence of semiclassical bound states of nonlinear Schrödinger equations with potentials of the class $(V)_{a}$, Comm. Partial Differential Equations 13 (1988), no. 12, 14991519. MR.0970154 (90d:35063a)

[Oh2] Stability of semiclassical bound states of nonlinear Schrödinger equations with potentials, Comm. Math. Phys. 121 (1989), no. 1, 11-33. MR0985612 (90b:35219)

[Oh3] _ On positive multi-lump bound states of nonlinear Schrödinger equations under multiple well potential, Comm. Math. Phys. 131 (1990), no. 2, 223-253. MR.1065671|(92a:35148)

[P] B. Plohr, Ph. D. Thesis, Princeton, 1978.

[R] W. G. Ritter, Gauge theory: instatons, monopoles, and moduli spaces, Preprint, arXiv:mathph/0304026 v1, 2003.

[Riv1] T. Rivière, Ginzburg-Landau vortices: the static model, Séminaire Bourbaki, Vol. 1999/2000, Astérisque No. 276 (2002), 73-103. MR.1886757 (2003f:58031)

[Riv2] Towards Jaffe and Taubes conjectures in the strongly repulsive limit, Manuscripta Math. 108 (2002), 217-273. MR1918588 (2003j:35105)

[Rub1] J. Rubenstein, On the equilibrium position of Ginzburg-Landau vortices, Z. Angew. Math. Phys. 46 (1995), no. 5, 739-751. MR1355536 (97d:82089)

[Rub2] Six lectures on superconductivity, Boundaries, Interfaces, and Transitions (Banff, AB, 1995), CRM Proc. Lecture Notes, vol. 13, Amer. Math. Soc., Providence, RI, 1998, pp. 163184. MR $1619115(99 \mathrm{e}: 82101)$ 
[RS] M. Reed and B. Simon, Methods of modern mathematical physics. Vols. I-IV, Academic Press, New York etc., 1972-1979. MF0493419 (58:12429a)

[ST] I. M. Sigal and F. Ting, Stability of pinned vortices (to appear).

[T] M. Tinkham, Introduction to superconductivity, McGraw-Hill, New York, 1975.

Department of Mathematics, University of Toronto, Toronto, ON M5S 3G3, Canada

$U R L$ : www.math.toronto.edu/sigal

Department of Mathematics, University of Notre Dame, Notre Dame, IN 46556-4618

Current address: Department of Mathematical Sciences, Lakehead University, Thunder Bay, ON P7B 5E1, Canada

E-mail address: fridolin.ting@lakeheadu.ca

Received 20/NOV/2003

Originally published in English 